\title{
Microstructure of strongly sheared suspensions and its impact on rheology and diffusion
}

\author{
By JOHN F. BRADY AND JEFFREY F. MORRIS $\dagger$ \\ Division of Chemistry and Chemical Engineering, California Institute of Technology, \\ Pasadena, CA 91125, USA \\ e-mail: jfbrady@caltech.edu; jeff.morris@che.gatech.edu
}

(Received 7 August 1996 and in revised form 19 February 1997)

The effects of Brownian motion alone and in combination with an interparticle force of hard-sphere type upon the particle configuration in a strongly sheared suspension are analysed. In the limit $P e \rightarrow \infty$ under the influence of hydrodynamic interactions alone, the pair-distribution function of a dilute suspension of spheres has symmetry properties that yield a Newtonian constitutive behaviour and a zero self-diffusivity. Here, $P e=\dot{\gamma} a^{2} / 2 D$ is the Péclet number with $\dot{\gamma}$ the shear rate, $a$ the particle radius, and $D$ the diffusivity of an isolated particle. Brownian diffusion at large $P e$ gives rise to an $O\left(a P e^{-1}\right)$ thin boundary layer at contact in which the effects of Brownian diffusion and advection balance, and the pair-distribution function is asymmetric within the boundary layer with a contact value of $O\left(P e^{0.78}\right)$ in pure-straining motion; non-Newtonian effects, which scale as the product of the contact value and the $O\left(a^{3} P e^{-1}\right)$ layer volume, vanish as $P e^{-0.22}$ as $P e \rightarrow \infty$.

If, however, particles are maintained at a minimum separation of $2 b$, with $b>a$, by a hard-sphere force there is also a boundary layer of thickness of $O\left(a P e^{-1}\right)$, but the asymmetry of the pair-distribution function for this situation is $O(P e)$, with an excess of particles along the compressional axes. The product of the asymmetric pair-distribution function and the thin boundary layer volume is now $O(1)$ (with dependence on $b / a)$ as $P e \rightarrow \infty$, thus yielding non-Newtonian rheology with normal stresses scaling as $\eta \dot{\gamma}$, where $\eta$ is the fluid viscosity. For a dilute suspension without hydrodynamic interactions in a general linear flow, the bulk stress resulting from pair interactions is proportional to $\eta \dot{\gamma} \phi_{b}^{2}(a / b)$, where $\phi_{b}=\frac{4}{3} \pi b^{3} n$ is the thermodynamic volume fraction. Including hydrodynamic interactions, the hydrodynamic normal stress differences are $O\left(\eta \dot{\gamma} \phi^{2}\right)$. The $O\left(\phi^{2}\right)$ hydrodynamic contribution to the viscosity due to the boundary layer is shear-thickening. The broken symmetry and boundarylayer structure also yield a shear-induced self-diffusivity of $O\left(\dot{\gamma} a^{2} \phi\right)$ as $P e \rightarrow \infty$.

At higher concentrations the boundary-layer structure is the same, with the pairdistribution function outside the boundary layer changed from its dilute value to a concentration-dependent function $g^{\infty}(\boldsymbol{r} ; \phi)$, which must be determined selfconsistently; the function $g^{\infty}(\boldsymbol{r} ; \phi)$ is not determined here. The appropriate Péclet number at high concentration is based on the concentration-dependent short-time selfdiffusivity $\overline{P e}=\dot{\gamma} a^{2} / 2 D_{0}^{s}(\phi)$. The stress contributions from the boundary layer scale as $\eta \dot{\gamma} \phi^{2} g^{\infty}(2 ; \phi) D / D_{0}^{s}(\phi)$, where $g^{\infty}(2 ; \phi)$ is the pair-distribution function at contact, and are argued to be dominant at high concentrations. The long-time self-diffusivity arising from the boundary-layer structure is predicted to scale as $\dot{\gamma} a^{2} \phi g^{\infty}(2 ; \phi)$.

$\dagger$ Present address: School of Chemical Engineering, Georgia Institute of Technology, Atlanta, GA 30332, USA. 


\section{Introduction}

This work addresses the microstructure of low-Reynolds-number suspensions in strong shearing flow where the influence of Brownian motion is weak. We have analysed the pair-distribution function under the combined influence of weak Brownian motion and an interparticle force of hard-sphere type. The study is motivated by a number of observations in strongly sheared suspensions. When particles are added to a Newtonian solvent, the suspension is often non-Newtonian, and thus the particles cause a more striking alteration of rheological properties than the simple and familiar increase in the effective viscosity. The effects in suspensions of spherical particles include normal stress differences, which have been observed experimentally (Gadala-Maria 1979) and in simulations (Phung, Brady \& Bossis 1996; Phung 1993), and a contribution to the isotropic stress (or particle pressure), which has been described and determined in simulation (Jeffrey, Morris \& Brady 1993). It is therefore generally inappropriate to apply Newtonian constitutive relations in modelling suspension flows, as this can yield predictions of velocity and particle fraction fields in disagreement with experiment for flows far from equilibrium. Far from equilibrium here implies large values of the Péclet number, $P e=\dot{\gamma} a^{2} / 2 D$, which is a measure of the relative strength of shear to Brownian forces. Here, $\dot{\gamma}$ is the magnitude of the shear rate, $a$ the particle size, and $D=k T / 6 \pi \eta a$ is the diffusivity of an isolated particle with thermal energy $k T$ in a fluid of viscosity $\eta$. Constitutive modelling of suspensions for arbitrary flows requires understanding the microscopic basis for bulk rheological behaviour at all values of $P e$. It is the goal of this work to analytically determine the microstructure of a strongly sheared suspension and demonstrate certain consequences of the microstructure for suspension rheology and self-diffusivity.

The rheology of suspensions is related to the spatial distribution of particles, or the microstructure, and qualitative features of the rheology may be ascribed to symmetry properties of the microstructure. For a suspension of purely hydrodynamically interacting spheres in the limits of $P e=0$ and $P e^{-1}=0$, the pair-distribution function possesses 'fore-aft' symmetry. Fore-aft symmetry is characterised by equal probability of a second particle lying on a trajectory where it is approaching (fore) or receding (aft) relative to a reference particle, and is relevant for flows in which the approaching and receding portions of the purely hydrodynamic pair trajectory are mirror images. This is true of simple-shear and planar (two-dimensional) extensional flow. If fore-aft symmetry of $g(\boldsymbol{r})$ holds, the rheology is Newtonian in the sense that normal stress differences and the suspension pressure are zero. This work will demonstrate how the combined effects of Brownian motion and an interparticle force give rise to the microstructural asymmetry necessary to yield non-Newtonian behaviour at large $P e$. Broken fore-aft symmetry at $P e^{-1}=0$ is also reflected in a finite longtime self-diffusivity, which we calculate using the theory of self-diffusivity in sheared suspensions developed in Morris \& Brady (1996).

The analytical study of the pair-distribution function in flowing suspensions was initiated by Batchelor \& Green (1972b) who showed that under the action of hydrodynamic interactions alone in pure straining flow, at $P e^{-1} \equiv 0, g(\boldsymbol{r})$ is spherically symmetric. This is a remarkable symmetry considering the angular dependence of the relative velocity of two particles, whereas isotropic microstructure at equilibrium, $P e \equiv 0$, is expected. In simple-shear flow there is a region of closed trajectories, and therefore one cannot conclude that $g(\boldsymbol{r})$ is isotropic, although this is a possible structure. At finite $P e$, flow distorts $g$ from spherical symmetry as shown by Batchelor $(1977)$ in his study of a weakly strained $(P e \ll 1)$ suspension, although his determination of $g$ to $O(P e)$ was insufficient to calculate non-Newtonian effects. To determine 
the effect of flow upon the normal stresses requires the next correction to $g$, which is $O\left(\mathrm{Pe}^{2}\right)$ (Brady \& Vicic 1995). Numerous studies of the pair-distribution function at small Pe have followed Batchelor's (1977) work, most with the aim of incorporating many-body effects present in suspensions at higher concentrations.

At equilibrium, the probability of a particular configuration is proportional to the Boltzmann factor $\exp [-V]$, where $V$ is the potential energy for the configuration made dimensionless by the thermal energy $k T$. In a flowing suspension, however, the interplay of Brownian motion, interparticle forces, and hydrodynamic interactions leads to a non-equilibrium microstructure that can only be determined analytically by solution of the Smoluchowski equation governing the configurational probability. The primary factor determining the microstructure in a flowing suspension is the relative strength of the shear-driven flux to the diffusive Brownian flux, i.e. Pe. Suspended particles are often of micron size or larger, which for typical shear rates yields $P e>O(1)$, and the microstructure is determined predominantly by hydrodynamic effects.

The effect of flow at large Péclet number upon the pair-distribution function has been demonstrated by experiment and simulation. Figure 1, adapted from Phung (1993), and figure 2, reproduced from Parsi \& Gadala-Maria (1987), are plots of the steady $g(\boldsymbol{r})$ found at large values of $P e$. Phung determined $g$ from Stokesian Dynamics (Brady \& Bossis 1988) simulation of a suspension in shear flow $u_{x}=\dot{\gamma} y$; the projections of $g$ onto the plane of shear, $x, y$, and the plane perpendicular to the flow, $y, z$, are shown. These were fully three-dimensional simulations at zero Reynolds number of a monodisperse suspension of spheres at a particle volume fraction of $\phi=0.45$; there were no interparticle forces. The pair-distribution function from nearequilibrium, $P e=0.01$, is presented for purposes of comparison with that for $P e=10^{4}$. At $P e=10^{4}$, there is narrowing of the nearest-neighbour ring in both projections relative to that at $P e=0.01$, and an obvious distortion of this ring from circular in the plane of shear. Parsi \& Gadala-Maria (1987) performed simple-shear flow experiments with a suspension of polystyrene spheres of radius $20-25 \mu \mathrm{m}$ in silicone oil at $\phi=0.4$, $P e=3.0 \times 10^{5}$ and $R e=3.2 \times 10^{-7}$. Colloidal forces are typically considered negligible for particles of this size. The pair-distribution function, determined by analysis of video images of particle positions, is illustrated by the projection of $g$ in the plane of shear in figure 2. The pair-distribution functions determined by Parsi \& Gadala-Maria and Phung have the common feature of an excess of particle pairs along the compressional axes and relatively small values in the extensional quadrants. With flow reversal, the compressional and extensional quadrants are interchanged and, as demonstrated by the results of Parsi \& Gadala-Maria, the fore-aft asymmetry is reversed after a period of microstructural rearrangement that scales with the applied strain.

Normal stress differences resulting from the asymmetry of $g(\boldsymbol{r})$ have been observed in strongly sheared suspensions both experimentally by Gadala-Maria (1979) and in Stokesian Dynamics simulation by Phung et al. (1996) and Phung (1993). In a parallel-plate geometry using suspensions identical to those of Parsi \& GadalaMaria (1987), Gadala-Maria found normal stress differences that scaled roughly as $\eta \dot{\gamma}$ for $\phi=0.3-0.5$. In suspensions of Brownian particles interacting only through hydrodynamics, Phung et al. reported normal stress differences for a suspension of $\phi=0.45$ at values of $P e$ up to $10^{5}$, and again the stress differences scaled hydrodynamically as $\eta \dot{\gamma}$ for large $P e$. Since interparticle forces are absent in these simulations, the results demonstrate that residual Brownian diffusion creates sufficient microstructural asymmetry to yield measurable non-Newtonian effects for $P e \gg 1$. Further experimental evidence of the asymmetry of $g(\boldsymbol{r})$ at large $P e$ is given by the 

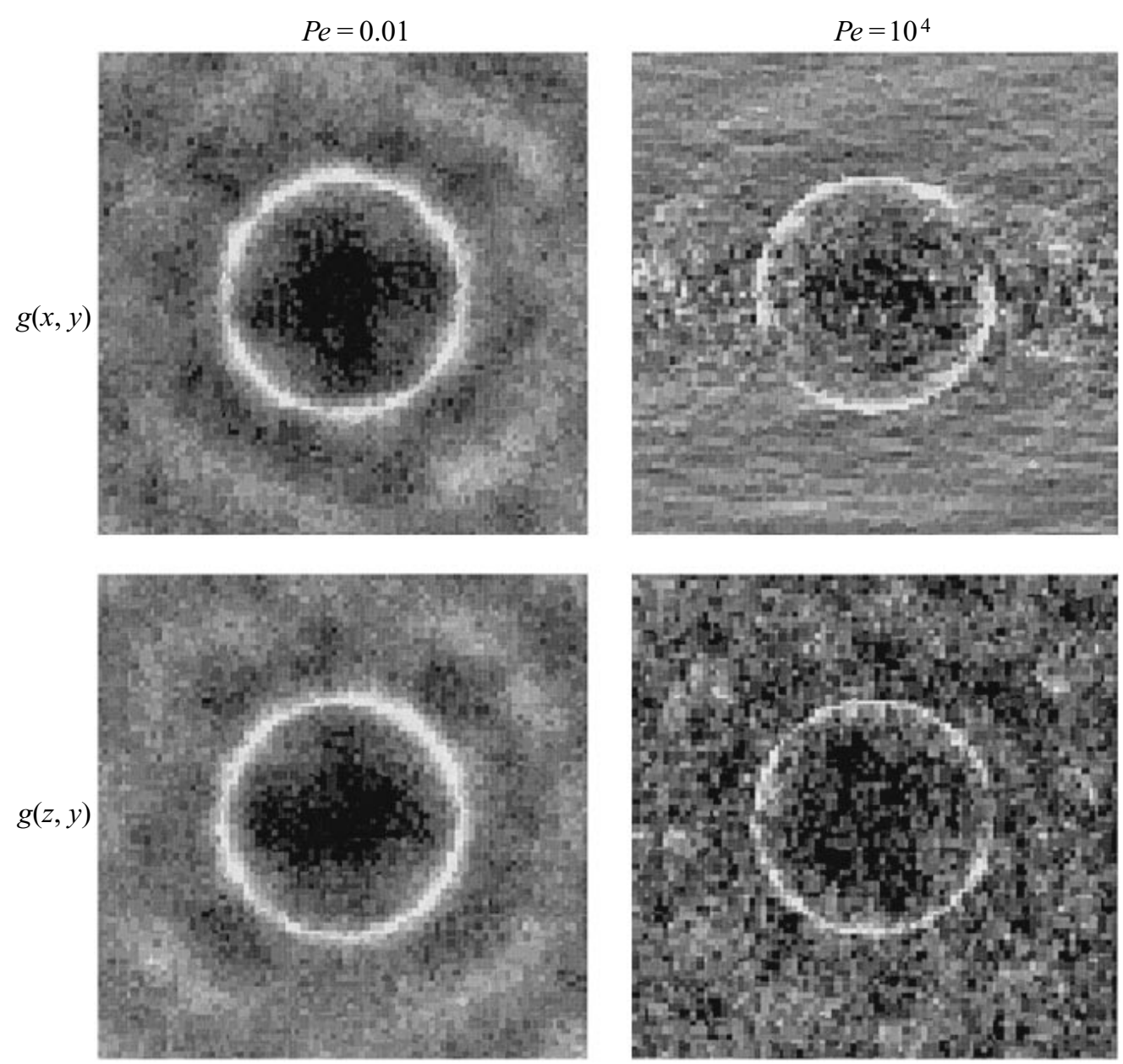

FIGURE 1. Projections of the pair-distribution function $g$ onto the $(x, y)$ - and $(y, z)$-planes for a monodisperse suspension at $\phi=0.45$ in simple shear, $u_{x}=\dot{\gamma} y$, simulated by Stokesian Dynamics. Light regions are high probability and dark low. There are 64 particles in the unit cell. Note the distortion in the nearest-neighbour ring in the $(x, y)$-plane and the narrowing of this ring in both the $(x, y)$ - and $(y, z)$-planes for $P e=10^{4}$ relative to $P e=0.01$. From Phung (1993).

work of Gadala-Maria \& Acrivos (1980), where for suspensions of $\phi>0.3$ the torque required to generate an oscillatory torsional flow in a parallel-plate viscometer went through a marked transient before reaching steady state at each reversal of the flow direction.

These examples are evidence of non-Newtonian behaviour in suspensions at large Péclet number, and what is very surprising is that the asymmetry persists at very high $\mathrm{Pe}$ where one would expect the effects of Brownian motion to be weak. Further, when Brownian motion and interparticle forces vanish, it has been shown analytically for dilute suspensions that the microstructure is symmetric and the rheology Newtonian. Considering only Stokes flow, we shall term the condition where Brownian motion and interparticle forces are absent the 'pure-hydrodynamic limit'. A simple argument based upon the reversibility of Stokes flow requires that fore-aft symmetry hold also for non-dilute suspensions in the pure-hydrodynamic limit. Thus, the experimental and simulation results seem to contradict the analytical reasoning. 
(a) $\dot{\gamma}>0$, steady state
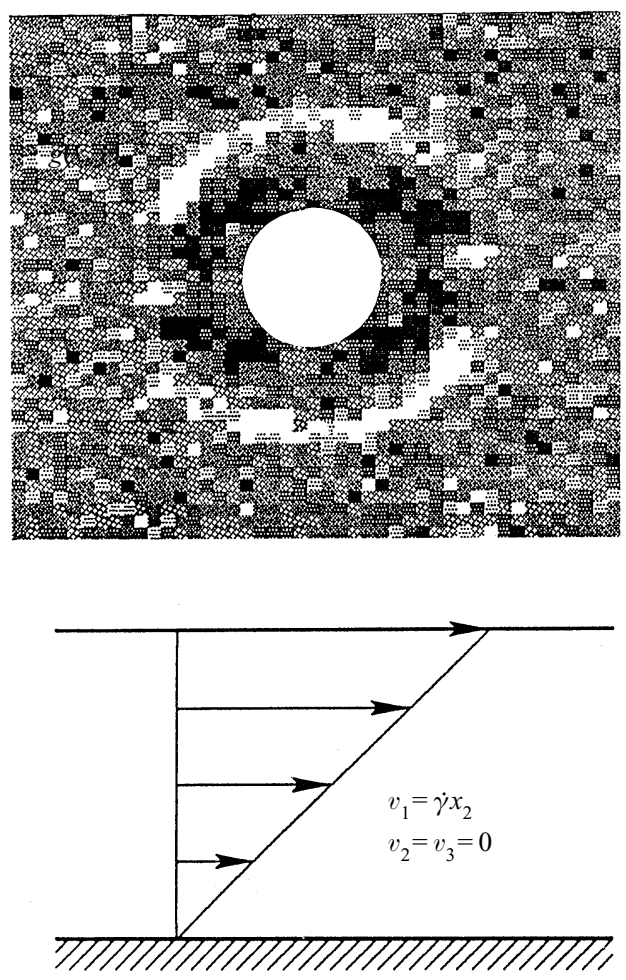

(b) $\dot{\gamma}<0$, steady state
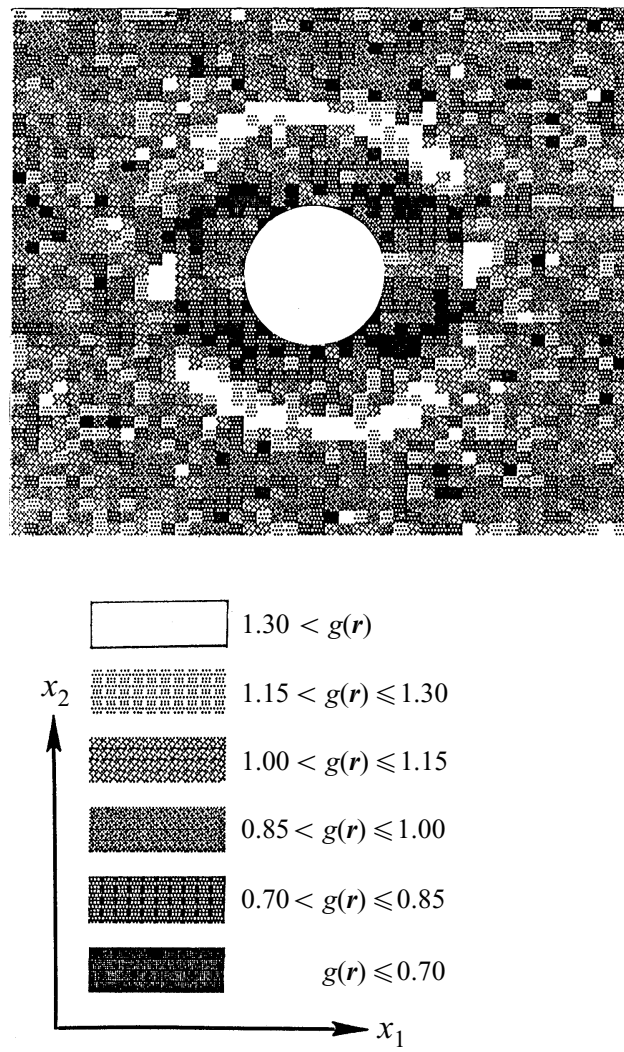

FIGURE 2. Pair-distribution function $g$ in the plane of shear for a suspension of polystyrene spheres in silicone oil at particle volume fraction $\phi=0.4$ in simple shear at $P e=3.0 \times 10^{5}$ and $R e=3.2 \times 10^{-7}$ The shear rate is opposite in the two plots. Note the fore-aft asymmetry of the pair distribution and the reversal of the asymmetry for reversal of the shear rate. From Parsi \& Gadala-Maria (1987).

To clarify this situation and to predict the rheology of non-colloidal suspensions, we have analysed the pair-distribution function of a monodisperse suspension of spheres for weak Brownian motion $(P e \gg 1)$. Under these conditions, there is a balance between advection and Brownian diffusion in a narrow $O\left(a \mathrm{Pe}^{-1}\right)$ boundary layer at minimum separation, analogous to boundary layers encountered in heat or mass transfer from surfaces, and it is within the boundary layer that the symmetry properties of $g$ are determined. Two cases distinguished by the minimum separation of a pair of particles, denoted $2 b$ so that $b$ is the effective radius of a particle, have been studied. In the first case, a repulsive force of hard-sphere type maintains a minimum separation of $2 b>2 a$. In the second case, the particles interact only hydrodynamically, and the minimum separation is at actual contact, i.e. $b=a$.

We first study the steady pair equation for dilute concentrations considering only pair interactions. In $\S 3$ and $\S 4$, we analyse the influence of a repulsive interparticle force of hard-sphere type by considering particles maintained at a minimum separation $2 b>2 a$. Fundamental features of the microstructure are the same regardless of whether the particles interact hydrodynamically: the radial balance between advection and Brownian diffusion yields an asymmetric $g(\boldsymbol{r})$ of $O(P e)$ within the boundary layer, and in the limit $\mathrm{Pe} \rightarrow \infty$, the product of the $O(P e)$ asymmetry and the 
$O\left(a^{3} P e^{-1}\right)$ boundary-layer volume is finite, yielding non-Newtonian rheology that is independent of $\mathrm{Pe}$. To demonstrate most clearly the boundary-layer balance, we first present the analysis neglecting hydrodynamics in $\$ 3$. The non-Newtonian behaviour of the suspension is illustrated by dilute-limit calculations of normal stress differences, Trouton ratio, and suspension pressure for simple-shear and several pure straining flows. We also show that the same qualitative and quantitative features are obtained with a repulsive hard-sphere force and shearing flow, but in the complete absence of Brownian motion; the thin diffusive boundary layer is replaced by a surface of discontinuity, which leads to the same results as obtained with a small but finite amount of Brownian motion. Thus, the conclusions we reach are valid whether one considers the asymmetry to be caused by a small amount of Brownian motion, or by some other non-thermal effect.

Analysis including pair hydrodynamic interactions, presented in $\S 4$, shows that nonNewtonian effects scale as $(b / a-1)^{0.22}$ as $b-a \rightarrow 0$. Extensions of the analyses for $b>a$ to non-dilute $\phi$ are presented for the cases with and without hydrodynamics in $\S 3.3$ and $\S 4.3$, respectively. In each case, the boundary-layer structure remains the same at larger $\phi$, but the pair-distribution function must asymptote to a concentrationdependent and self-consistently determined value $g^{\infty}(\boldsymbol{r} ; \phi)$, rather than to its dilute value, outside the layer. (We have not analysed $g^{\infty}$.) Furthermore, the appropriate Péclet number is that based on the short-time self-diffusivity at the conditions of interest: $\overline{P e}=\dot{\gamma} a^{2} / 2 D_{0}^{s}(\phi)$.

To understand the approach to the symmetric microstructure and Newtonian rheology of the pure-hydrodynamic limit, we have analysed the pair-distribution function with hydrodynamics and weak Brownian motion, but no interparticle forces $(b \equiv a)(\S 5)$. Once again, there is a boundary layer of $O\left(a P e^{-1}\right)$ thickness in which $g(\boldsymbol{r})$ is asymmetric, and under these conditions, the boundary-layer equation governing $g$ for the case of pure straining flow is solved by similarity reduction to find $g(2)=$ $O\left(P e^{0.78}\right)$, where $g(2)$ is the contact value of $g(\boldsymbol{r})$. Thus, we find that non-Newtonian effects scale as $O\left(\mathrm{Pe}^{-0.22}\right)$ as $\mathrm{Pe} \rightarrow \infty$. The exponent -0.22 arises from a grouping of the contact values of hydrodynamic functions that also yields the $(b / a-1)^{0.22}$ scaling for short-ranged repulsive forces.

The asymmetry in the boundary-layer solution for $b>a$ is also reflected in an $O(\phi)$ cross-stream self-diffusivity in simple shear, which we calculate in $\$ 6$ for the case of hard spheres with and without hydrodynamics. We also show that the diffusivity may be interpreted as a mobility times an 'osmotic' pressure gradient driving force. At high concentrations we predict that the long-time self-diffusivity scales as $\dot{\gamma} a^{2} g^{\infty}(2 ; \phi)$. Finally, we discuss the implications of this work for the rheological and diffusive behaviour of suspensions and possible extensions of this work to other situations.

\section{Governing equations}

\subsection{Smoluchowski equation}

We consider a suspension of $N$ identical spheres homogeneously dispersed in a Newtonian fluid. The suspension is subjected to a linear incompressible flow with constant velocity-gradient tensor $\dot{\boldsymbol{\Gamma}}$. The probability distribution function for the $N$-particle configuration, $\boldsymbol{x}^{N}$, is denoted $P_{N}\left(\boldsymbol{x}^{N}, t\right)$ and satisfies the Smoluchowski equation

$$
\frac{\partial P_{N}}{\partial t}+\sum_{\alpha=1}^{N} \nabla_{\alpha} \cdot \boldsymbol{j}_{\alpha}=0
$$


with the flux of particle $\alpha$ given by

$$
\boldsymbol{j}_{\alpha}=\boldsymbol{U}_{\alpha} P_{N}-\sum_{\beta=1}^{N} \boldsymbol{D}_{\alpha \beta} P_{N} \cdot \nabla_{\beta}\left(\ln P_{N}+V\right),
$$

where $\boldsymbol{U}_{\alpha}$ is the hydrodynamic velocity, $V$ is the potential energy made dimensionless with $k T$, and the Brownian diffusivity is given by $\boldsymbol{D}_{\alpha \beta}=k T \boldsymbol{M}_{\alpha \beta}$, where $\boldsymbol{M}_{\alpha \beta}$ is the hydrodynamic mobility of particle $\alpha$ due to a force exerted on particle $\beta$. We take $V$ to be an interparticle potential depending only upon the relative configuration of the particles. A potential dependent upon absolute position, and hence the influence of particle buoyancy, for example, may be included without altering the basic formulation; the flux from such a potential is appropriately grouped with the hydrodynamic velocity because both generate a non-equilibrium microstructure.

Equation (1) is integrated with respect to the centre-of-mass coordinate of a pair of particles and the positions of the remaining $N-2$ particles to arrive at the equation for $P_{1 \mid 1}(\boldsymbol{r})$, the probability distribution for finding a particle at $\boldsymbol{r}$ given that a particle lies at the origin:

$$
\begin{aligned}
\frac{\partial P_{1 \mid 1}}{\partial t}+ & \nabla_{r} \cdot\left(\langle\boldsymbol{U}\rangle_{2} P_{1 \mid 1}\right)-\nabla_{r} \cdot\left[P_{1 \mid 1}\left\langle\boldsymbol{D} \cdot \nabla_{r}\left(\ln P_{N}+V\right)\right\rangle_{2}\right] \\
& -\nabla_{r} P_{1 \mid 1} \cdot \int P_{3 \mid 2}\left(\boldsymbol{x}_{3} \mid \boldsymbol{r}\right)\left\langle\left(\boldsymbol{D}_{13}-\boldsymbol{D}_{23}\right) \cdot \nabla_{3}\left(\ln P_{N}+V\right)\right\rangle_{3} d \boldsymbol{x}_{3}=0,
\end{aligned}
$$

where $\nabla_{r}=\nabla_{2}=-\nabla_{1}$, and $P_{3 \mid 2}$ is the probability of finding a third particle at $\boldsymbol{x}_{3}$ given the positions of two particles. In (3), the relative velocity and relative diffusivity are given by

$$
\boldsymbol{U}=\boldsymbol{U}_{2}-\boldsymbol{U}_{1}, \quad \text { and } \quad \boldsymbol{D}=\boldsymbol{D}_{11}-\boldsymbol{D}_{12}-\boldsymbol{D}_{21}+\boldsymbol{D}_{22},
$$

respectively, and \langle\rangle$_{2}$ indicates a conditional average with two particles fixed.

To make analytical progress, we consider dilute particle volume fraction, $\phi=$ $4 \pi n a^{3} / 3 \ll 1$, with $n$ the number density of particles. Thus, with an $O(\phi)$ error the nonlinear averages and integral over the position of a third particle in (3) are neglected. Quantities are made dimensionless by scaling as

$$
\boldsymbol{r} \sim a, \quad \boldsymbol{U} \sim \dot{\gamma} a, \quad \boldsymbol{D} \sim 2 D, \quad \text { and } t \sim \dot{\gamma}^{-1},
$$

where $\dot{\gamma}$ is a characteristic magnitude of the velocity gradient tensor $\dot{\boldsymbol{\Gamma}}$, and the scaling of the relative diffusivity is with its far-field asymptotic value of $2 D$, with $D=k T / 6 \pi \eta a$ the diffusivity of an isolated particle of radius $a$ and thermal energy $k T$ in a fluid of viscosity $\eta$. The Péclet number is defined by

$$
P e \equiv \frac{\dot{\gamma} a^{2}}{2 D}=\frac{3 \pi \eta a^{3} \dot{\gamma}}{k T} .
$$

In order to investigate the behaviour under the action of interparticle forces, we consider a simple hard-sphere interparticle force at a variable distance $r=2 b$. This force may be incorporated through a no-flux condition rather than in the flux itself. Thus, the dimensionless equation and associated boundary conditions governing the 
pair-distribution function $g(\boldsymbol{r})$, defined by $P_{1 \mid 1}(\boldsymbol{r})=n g(\boldsymbol{r})$, are

$$
\begin{aligned}
& \frac{\partial g}{\partial t}+\nabla \cdot(\boldsymbol{U} g)-P e^{-1} \nabla \cdot(\boldsymbol{D} \cdot \nabla g)=0, \\
& \hat{\boldsymbol{r}} \cdot\left(\boldsymbol{j}_{2}-\boldsymbol{j}_{1}\right)=0 \quad \text { at } \quad r=2 b / a, \\
& g \sim 1 \quad \text { as } \quad r \rightarrow \infty,
\end{aligned}
$$

where $\hat{\boldsymbol{r}}=\boldsymbol{r} / \boldsymbol{r}$ is the unit vector projecting from particle 1 to particle 2 . To simplify notation in $(5 a)$ and hereafter, we write $\nabla$ for $\nabla_{r}$ and the angle brackets indicating averaging are omitted. Setting $b \equiv a$ corresponds to no interparticle forces, and we shall refer to this case as 'pure hydrodynamics'.

We are interested in the limit $P e \gg 1$, for which the steady solution of $(5 a-5 c)$ over most of the domain is well-approximated by the solution to

$$
\nabla \cdot(\boldsymbol{U} g)=0,
$$

with the far-field condition of

$$
g \sim 1 \text { as } \quad r \rightarrow \infty .
$$

Neglecting the highest derivative in $(5 a)$ does not permit applying the no-flux boundary condition at contact. Batchelor \& Green (1972b) solved (6) with (7), demonstrating that when the trajectory of the second sphere relative to the reference sphere comes from infinitely far away, where it is assumed that all positions of the second sphere are equally likely, $g$ is a function of scalar separation only. The pair distribution under these conditions, denoted $g(\boldsymbol{r})=p(r)$, is

$$
p(r)=\frac{1}{1-A} \exp \left[\int_{r}^{\infty} \frac{3(B-A)}{r(1-A)} \mathrm{d} r\right] .
$$

The scalar functions $A(r)$ and $B(r)$ specify the radial dependence of the disturbance of the relative velocity from $\dot{\boldsymbol{\Gamma}} \cdot \boldsymbol{r}$ due to pair hydrodynamic interactions (Batchelor \& Green 1972a),

$$
\boldsymbol{U}-\dot{\boldsymbol{\Gamma}} \cdot \boldsymbol{r}=-\boldsymbol{r} \cdot \hat{\boldsymbol{E}} \cdot[A(r) \hat{\boldsymbol{r}} \hat{\boldsymbol{r}}+B(r)(\boldsymbol{I}-\hat{\boldsymbol{r}} \hat{r})],
$$

where $\hat{\boldsymbol{E}}$ is the dimensionless rate-of-strain tensor. The functions $A$ and $B$ may be expressed in terms of the standard mobility and resistance functions (see Kim \& Karrila 1991, pp. 276-277). Beyond its purely radial dependence, the main feature of note regarding $p$ is that it diverges at particle contact, as $(r / a-2)^{-0.78}$ with a logarithmic correction (Batchelor \& Green 1972b; see (40) below). This divergence is sufficiently weak that, while $p$ is not forced to satisfy the no-flux condition at the inner boundary, for pure hydrodynamics $(b \equiv a)$ it nevertheless does so because the radial velocity is linear in $r / a-2$ and thus $U_{r} p$ vanishes at contact.

The spherically symmetric solution for the pair-distribution function is only strictly valid when all particle trajectories come from infinity, as is the case in pure straining flow. In simple-shear flow, there are regions of closed trajectories, and there is no guarantee that $g(\boldsymbol{r})=p(r)$ in this region. Thus, the familiar problem of simple-shear flow requires special treatment. With repulsive interparticle forces of sufficient range $\left(b / a-1 \geqslant 10^{-4}\right)$, however, the closed streamline region is destroyed. The work of Smart \& Leighton (1989) on the influence of surface roughness suggests that $b / a-1$ should be larger than $10^{-3}$ in most practical situations. 


\subsection{Bulk stress}

We shall also need to compute the particle contribution to the bulk stress given by (Brady 1993a)

$$
\left\langle\Sigma_{p}\right\rangle=-n k T \boldsymbol{I}+n\left(\left\langle\mathbf{S}^{B}\right\rangle+\left\langle\boldsymbol{S}^{P}\right\rangle+\left\langle\mathbf{S}^{H}\right\rangle\right)
$$

where $n k T \boldsymbol{I}$ is the familiar kinetic contribution that yields the dilute osmotic pressure, and

$$
\begin{aligned}
& \left\langle\boldsymbol{S}^{B}\right\rangle=-k T\left\langle\nabla \cdot\left(\boldsymbol{R}_{S U} \cdot \boldsymbol{R}_{F U}^{-1}\right)\right\rangle, \\
& \left\langle\boldsymbol{S}^{P}\right\rangle=-\left\langle\left(\boldsymbol{x} \boldsymbol{I}+\boldsymbol{R}_{S U} \cdot \boldsymbol{R}_{F U}^{-1}\right) \cdot \boldsymbol{F}^{P}\right\rangle, \\
& \left\langle\boldsymbol{S}^{H}\right\rangle=-\left\langle\boldsymbol{R}_{S U} \cdot \boldsymbol{R}_{F U}^{-1} \cdot \boldsymbol{R}_{F E}-\boldsymbol{R}_{S E}\right\rangle:\langle\boldsymbol{E}\rangle,
\end{aligned}
$$

are, respectively, expressions for the Brownian, interparticle force, and hydrodynamic stresslets. The particle stress may be recast as

$$
\begin{aligned}
& \left\langle\Sigma_{P}\right\rangle=-n k T \boldsymbol{I}-n k T a \oint_{r=2 a} \hat{\boldsymbol{r}} \hat{\boldsymbol{r}} P_{1 \mid 1}(\boldsymbol{r}) d S-n\left\langle\boldsymbol{x} \boldsymbol{F}^{P}\right\rangle \\
& -n\left\langle\boldsymbol{R}_{S U} \cdot \boldsymbol{R}_{F U}^{-1} \cdot \boldsymbol{R}_{F E}-\boldsymbol{R}_{S E}\right\rangle:\langle\boldsymbol{E}\rangle+n k T\left\langle\boldsymbol{R}_{S U} \cdot \boldsymbol{R}_{F U}^{-1} \cdot \nabla\left[V+\ln P_{N}\right]\right\rangle,
\end{aligned}
$$

by decomposing $\left\langle\mathbf{S}^{B}\right\rangle$ into the contact integral and the final term involving $\nabla \ln P_{N}$. In (11), the subscripts on the resistance tensors $\boldsymbol{R}_{F U}$ and $\boldsymbol{R}_{S E}$ denote the relation of force to velocity and stress to rate of strain, respectively, and the others should be clear from these.

The bulk stress of the entire suspension - particles plus fluid - is

$$
\langle\Sigma\rangle=-\langle p\rangle_{f} \boldsymbol{I}+2 \eta\langle\boldsymbol{E}\rangle+\left\langle\Sigma_{p}\right\rangle,
$$

where $\langle p\rangle_{f}$ is the average pressure in the fluid phase, which for an incompressible fluid is arbitrary.

\section{Interparticle forces, no hydrodynamics}

\subsection{Pair-distribution function without hydrodynamics}

The singular nature of the high- $P e$ limit is most easily seen when hydrodynamic interactions are neglected. This corresponds to the case $b \gg a$, i.e. particles interacting by long-range repulsive forces of hard-sphere type. In the absence of hydrodynamic interactions, $\boldsymbol{U}=\dot{\boldsymbol{\Gamma}} \cdot \boldsymbol{r}$ and the pair diffusivity is a constant equal to $2 D$. The hydrodynamic radius, $a$, does not enter explicitly, and we scale distances with $b$ and define a new Péclet number

$$
P e_{b}=\dot{\gamma} b^{2} / 2 D=3 \pi a b^{2} / k T,
$$

in which the residual dependence on $a$ arises because the particle diffusivity is sensitive to the actual, or hydrodynamic, radius.

The pair-distribution function is governed by

$$
\begin{gathered}
\nabla^{2} g-P e_{b} \dot{\Gamma} \cdot \boldsymbol{r} \cdot \nabla g=0, \\
\frac{\partial g}{\partial r}=2 P e_{b} \gamma_{r} g \quad \text { at } \quad r=2, \\
g \sim 1 \quad \text { as } \quad r \rightarrow \infty .
\end{gathered}
$$

At large Péclet number, except near contact, $(12 a)$ reduces to $\dot{\boldsymbol{\Gamma}} \cdot \boldsymbol{r} \cdot \nabla g=0$, i.e. on a streamline $g$ is a constant, which $(12 c)$ dictates to be unity. (Note that this is the solution of (8) in the absence of hydrodynamic interactions, $A=B=0$.) 
This constant solution does not satisfy the no-flux condition at contact where there is a boundary layer in which the effects of Brownian motion balance those of advection. Thus, near contact we stretch $r$ as

$$
y=P e_{b}(r-2) .
$$

The governing equation and boundary conditions for $g$ in the stretched coordinates are

$$
\begin{gathered}
\frac{\partial^{2} g}{\partial y^{2}}-2 \gamma_{r}\left[1-\frac{1}{2 \gamma_{r}} P e_{b}^{-1}+\frac{1}{2} P e_{b}^{-1} y\right] \frac{\partial g}{\partial y}=P e_{b}^{-1}\left[\gamma_{\theta} \frac{\partial g}{\partial \theta}+\frac{\gamma_{\varphi}}{\sin \theta} \frac{\partial g}{\partial \varphi}\right]+O\left(P e_{b}^{-2}\right), \\
\frac{\partial g}{\partial y}=2 \gamma_{r} g \quad \text { at } y=0, \\
g \sim 1 \quad \text { as } \quad y \rightarrow \infty .
\end{gathered}
$$

We take $\theta$ as the azimuthal angle measured from the $x_{3}$-axis, and $\varphi$ as the polar angle measured from the $x_{1}$-axis. In $(12 a)-(13 b) \gamma_{r}, \gamma_{\theta}$, and $\gamma_{\varphi}$ are defined by

$$
\gamma_{r}=\hat{\boldsymbol{r}} \cdot \hat{\boldsymbol{\Gamma}} \cdot \hat{\boldsymbol{r}}=\hat{\boldsymbol{r}} \cdot \hat{\boldsymbol{E}} \cdot \hat{\boldsymbol{r}}, \quad \gamma_{\theta}=\hat{\boldsymbol{\theta}} \cdot \hat{\boldsymbol{\Gamma}} \cdot \hat{\boldsymbol{r}}, \quad \gamma_{\varphi}=\hat{\boldsymbol{\varphi}} \cdot \hat{\boldsymbol{\Gamma}} \cdot \hat{\boldsymbol{r}},
$$

where $\hat{\boldsymbol{\theta}}$ and $\hat{\boldsymbol{\varphi}}$ are the unit vectors in the $\theta$ - and $\varphi$-directions, respectively, and $\hat{\boldsymbol{\Gamma}}$ is the non-dimensional velocity gradient tensor.

At leading-order in $P e_{b}^{-1}$ we have the balance

$$
\frac{\partial^{2} g}{\partial y^{2}}-2 \gamma_{r} \frac{\partial g}{\partial y}=0
$$

with general solution

$$
g(y)=1+\text { constant } \times \int_{y}^{\infty} \mathrm{e}^{\gamma_{r} z} \mathrm{~d} z
$$

which asymptotes to unity as $y \rightarrow \infty$. However, this solution does not satisfy the boundary condition at $y=0$, and thus terms of $O\left(P e_{b}^{-1}\right)$ must be retained in the equation for $g$. This failure may be explained by noting that the leading-order governing equation is the radial derivative of the boundary condition at $y=0$, so that either the equation or the boundary condition is redundant.

It is the competition between radial advection and diffusion that generates the large gradients in $g$ characteristic of the boundary layer, which prompts us to retain only those terms of $O\left(\mathrm{Pe}_{b}^{-1}\right)$ that appear in the divergence of the radial flux. That is, we solve the 'radial balance' obtained by discarding the right-hand side of $(13 a)$. The solution,

where $s(z)$ is given by

$$
g(y)=\frac{1+2 \gamma_{r} \int_{0}^{y} \mathrm{e}^{s(z)} \mathrm{d} z}{1+2 \gamma_{r} \int_{0}^{\infty} \mathrm{e}^{s(z)} \mathrm{d} z},
$$

$$
s(z)=2 \gamma_{r}\left[\left(1-\frac{1}{2 \gamma_{r}} P e_{b}^{-1}\right) z+\frac{1}{4} P e_{b}^{-1} z^{2}\right],
$$

is valid only for $\gamma_{r}<0$. In the 'extensional quadrants', where $\gamma_{r}>0$, g remains $O(1)$; 
there is no boundary layer. Rewriting $g$ as

$$
g(y)=g(0)\left[1+2 \gamma_{r} \int_{0}^{y} \mathrm{e}^{s(z)} \mathrm{d} z\right],
$$

with

$$
g(0)=-\frac{4}{3} P e_{b} \gamma_{r}+O(1) \quad \text { as } \quad P e_{b} \rightarrow \infty,
$$

shows that there is an $O\left(P e_{b}\right)$ excess of particles in the 'compressional quadrants'.

Physically, the hard-sphere repulsion at $r=2 b$ acts as a strainer for particle centres, but permits the fluid to pass through unimpeded. On the compressive side the fluid velocity is directed radially inward $\left(\gamma_{r}<0\right)$ and a large $O\left(P e_{b}\right)$ buildup of particles occurs so that the outward diffusive flux can balance the inward advective flux. On the extensional side $\left(\gamma_{r}>0\right)$, the advective flux is directed radially outward, particle centres are not strained by the hard-sphere force, and no buildup occurs, i.e. there is no boundary layer and the variation in $g(\boldsymbol{r})$ remains $O(1)$. The situation is similar to heat transfer at high Péclet number with suction $\left(\gamma_{r}<0\right)$ or injection $\left(\gamma_{r}>0\right)$ at the boundary.

\subsection{Macroscopic stress without hydrodynamics}

The large $O\left(P e_{b}\right)$ buildup of particles in the thin $O\left(P e_{b}^{-1}\right)$ boundary layer leads to both shear and normal stresses that are independent of $P e_{b}$ in the limit $P e_{b} \rightarrow \infty$. The influence of a hard-sphere force for arbitrary $b \geqslant a$ is given by a 'contact' integral identical in form to that in (11) with $b$ replacing $a$; the hard-sphere force is a delta function at $r=2 b$. For $b>a$, or for $b=a$ without hydrodynamics, this integral represents the non-hydrodynamic hard-sphere stress, rather than a hydrodynamic Brownian stress. In the absence of hydrodynamics, $\left\langle\Sigma_{P}\right\rangle$ reduces to

$$
\left\langle\Sigma_{P}\right\rangle=-n k T\left[\boldsymbol{I}+\phi_{b} \frac{3}{\pi} \oint \hat{\boldsymbol{r}} \hat{\boldsymbol{r}} g(2 b \hat{\boldsymbol{r}}) \mathrm{d} \Omega\right]+5 \eta \phi\langle\boldsymbol{E}\rangle,
$$

where $5 \eta \phi\langle\boldsymbol{E}\rangle=2 \eta_{E}\langle\boldsymbol{E}\rangle$ is the shear stress due to the Einstein viscosity correction, $\eta_{E}=\frac{5}{2} \eta \phi$

The particle pressure is defined mechanically as

$$
\Pi=-\frac{1}{3} \boldsymbol{I}:\left\langle\Sigma_{P}\right\rangle,
$$

so that in equilibrium (18) yields the familiar hard-sphere fluid equation of state (see e.g. Hansen \& McDonald 1986),

$$
\frac{\Pi}{n k T}=1+4 \phi_{b} g(2 b) .
$$

We shall denote as $\Sigma^{\prime}$ the extra stress due to particle interactions over and above the equilibrium osmotic pressure and the Einstein viscosity. Inserting the contact value of $g(\boldsymbol{r})$ given by (17) into (18) yields

$$
\left\langle\Sigma^{\prime}\right\rangle=\eta \dot{\gamma} \phi_{b}^{2} \frac{a}{b} \frac{9}{\pi} \int_{\gamma_{r}<0} \hat{\boldsymbol{r}} \hat{\boldsymbol{r}} \gamma_{r} \mathrm{~d} \Omega+O\left(\eta \dot{\gamma} \phi_{b}^{2} \frac{a}{b} P e_{b}^{-1}\right),
$$

for a dilute suspension.

The boundary-layer analysis and (19) apply for any linear flow; because we have retained only the radial balance, only the rate-of-strain tensor appears in $\gamma_{r}$ and $\left\langle\Sigma^{\prime}\right\rangle$. As examples, we evaluate (19) for several basic flows. The first is simple-shear flow with velocity in the 1-direction and velocity gradient in the 2-direction, i.e. 
$E_{i j}=\frac{1}{2} \dot{\gamma}\left(\delta_{i 1} \delta_{j 2}+\delta_{i 2} \delta_{j 1}\right)$, which is also the rate of strain in planar extensional flow. The relative shear viscosity is

$$
\eta_{r}=1+\frac{5}{2} \phi+\frac{6}{5} \phi_{b}^{2} \frac{a}{b}
$$

the second normal stress difference is

$$
N_{2}=\left\langle\Sigma_{22}^{\prime}-\Sigma_{33}^{\prime}\right\rangle=-\eta \dot{\gamma} \phi_{b}^{2} \frac{a}{b} \frac{12}{5 \pi},
$$

and the osmotic pressure contribution from the flow is

$$
\Pi^{\prime}=\eta \dot{\gamma} \phi_{b}^{2} \frac{a}{b} \frac{4}{\pi} .
$$

Because of the symmetry along the compressional axes, the first normal stress difference, $N_{1}=\left\langle\Sigma_{11}^{\prime}-\Sigma_{22}^{\prime}\right\rangle$, vanishes.

In uniaxial extensional flow along the 3-direction, i.e. $E_{i j}=\dot{\epsilon}\left(\delta_{i 3} \delta_{j 3}-\frac{1}{2} \delta_{i 1} \delta_{j 1}\right.$ $-\frac{1}{2} \delta_{i 2} \delta_{j 2}$ ), where $\dot{\epsilon}$ is the extension rate, we have

and the osmotic pressure is

$$
\left\langle\Sigma_{33}^{\prime}\right\rangle=-\eta \dot{\epsilon} \phi_{b}^{2} \frac{a}{b} \frac{4}{5 \sqrt{3}}
$$

$$
\Pi^{\prime}=\eta \dot{\epsilon} \phi_{b}^{2} \frac{a}{b} \frac{4}{\sqrt{3}} .
$$

The 11 and 22 stresses are equal, and the Trouton ratio is given by

$$
\left\langle\Sigma_{33}^{\prime}\right\rangle-\frac{1}{2}\left(\left\langle\Sigma_{11}^{\prime}\right\rangle+\left\langle\Sigma_{22}^{\prime}\right\rangle\right)=\eta \dot{\epsilon} \phi_{b}^{2} \frac{a}{b} \frac{24}{5 \sqrt{3}} .
$$

The stresses for equi-biaxial extension are just twice those for uniaxial extension.

In both shear and extensional flows the high-Péclet-number viscosities are less than the corresponding viscosities at low Péclet number - the suspension shear thins. The low-shear-rate stress behaviour is Newtonian, and the viscosity from pair hard-sphere interactions in the absence of hydrodynamics is given by (Russel, Saville \& Schowalter 1989)

$$
\eta^{H S}\left(P e_{b}=0\right)=\eta \phi_{b}^{2} \frac{a}{b} \frac{12}{5} .
$$

Thus, in simple-shear flow the shear viscosity falls to half, while in extensional flow the Trouton ratio falls to $2 / 3 \sqrt{3}$, of their zero-shear-rate values.

In obtaining (15), we have neglected the $O\left(P e_{b}^{-1}\right)$ terms on the right-hand side of $(13 a)$ while retaining terms of the same magnitude in the radial balance on the left-hand side. This is generally not valid, and the right-hand side does affect the angular structure of $g(2)$. The $O\left(P e_{b}\right)$ magnitude of $g(2)$ is not affected, however. We have not been able to find a similarity transformation that would reduce $(13 a)$ to a set of ordinary differential equations. Solving the boundary-layer equation numerically is actually more difficult than solving the full equation for arbitrary Péclet number, as $(13 a)$ must be solved for large values of $P e_{b}$. Recent unpublished work by J. F. Brady \& M. A. Vicic (1997) solving (12a) for all $P e_{b}$ shows the boundary-layer structure exhibited by (15). The stress predictions from the radial balance for simple-shear flow are within $9 \%$ for all quantities except the first normal stress difference. The precise symmetry about the compressional axis is broken by the rotation of the simple-shear flow, $\left\langle\boldsymbol{\Sigma}_{11}^{\prime}\right\rangle$ increases (becomes less 
negative), and finite positive $\left(N_{1} \approx-\frac{1}{2} N_{2}\right)$ first normal stress differences occur as $P e_{b} \rightarrow \infty$.

Although we have considered a small amount of Brownian motion to generate an asymmetric structure in the limit of $P e \rightarrow \infty$, we would now like to show that the same results apply even in the complete absence of any thermal effects. Consider the case of two particles in simple-shear flow without hydrodynamics interacting through a repulsive force that gives an excluded shell at $r=2 b$, as discussed in Russel et al. (1989). On the compressive axes $\gamma_{r}<0$, the magnitude of the repulsive force must balance the advective radial velocity so that there is no flux at contact, i.e.

$$
\boldsymbol{F}=-6 \pi \eta \dot{\gamma} a \hat{\boldsymbol{r}} \cdot \hat{\boldsymbol{E}} \cdot \hat{\boldsymbol{r}} \boldsymbol{r} \quad \text { at } \quad r=2 b
$$

on the extensional axes there is no repulsive force. Without Brownian motion the excluded shell generates a discontinuity in the pair-distribution function at contact such that

$$
g(\boldsymbol{r})=H(r-2 b)+\frac{2 b}{3} \delta(r-2 b), \quad \gamma_{r}<0, \quad r \rightarrow 2 b,
$$

where $H$ is the Heaviside function and $\delta$ the delta function. Using this distribution and the force at contact from (20) in the expression for the stress from interparticle forces, and noting that the delta-function contribution gives $1 / 2$ because it is evaluated at the end point $\dagger$, we obtain precisely (19) from the stress due to the excluded shell. Thus, whether the asymmetry is broken by Brownian motion or a repulsive force and no thermal effects, the results are the same. Brownian motion is the proper way to smooth out the discontinuous distribution given by (21). It should also be clear that the same qualitative results would be obtained if the repulsive forces were long-ranged, as opposed to hard-sphere like.

\subsection{Extension to higher concentrations}

Since the important physics that breaks flow reversal symmetry and leads to finite $O(1)$ stresses at infinite Péclet number occurs in a thin boundary layer at particle contact, the analysis above can be extended to all concentrations. The only change is that rather than the pair-distribution function approaching unity outside the boundary layer, it must now asymptote to the surface value of the concentration-dependent pairdistribution function obtained by solving the purely advective problem, i.e. setting $P e_{b}^{-1} \equiv 0$ and ignoring the no-flux boundary condition at particle contact. We denote this distribution function by $g^{\infty}(\boldsymbol{r})$. It is not known how this function depends on $\phi_{b}$. For example, the equilibrium pair-distribution function at contact, $g^{e q}\left(r=2 b, \phi_{b}\right)$, diverges in the disordered state as $\left(1-\phi_{b} / \phi_{b m}\right)^{-1}$ as $\phi_{b} \rightarrow \phi_{b m}$, where $\phi_{b m} \approx 0.64$ (Hansen \& McDonald 1986). One might expect a similar divergence in $g^{\infty}$ from the geometric constraint of packing particles, but perhaps at a different maximum concentration. Nevertheless, the proceeding analysis applies, and in place of (19) we have, in the radial balance approximation,

$$
\left\langle\Sigma^{\prime}\right\rangle=\eta \dot{\gamma} \phi_{b}^{2} \frac{a}{b} \frac{9}{\pi} \int_{\gamma_{r}<0} \hat{\boldsymbol{r}} \hat{\boldsymbol{r}} g^{\infty}\left(2 b ; \phi_{b}\right) \gamma_{r} \mathrm{~d} \Omega+O\left(\eta \dot{\gamma} P e_{b}^{-1}\right) .
$$

The argument leading to (22) only considers the contribution from the asymmetric structure in the boundary layer. It is likely (if not certain) that there will be 'wake' regions originating at 'separation' points near the axes at which the radial velocity

$\dagger$ Russel et al. (1989) have twice the value given here because they did not take into account this endpoint effect. 
vanishes $\left(\gamma_{r}=0\right)$ that will advect the boundary-layer structure into the bulk of the suspension, rendering the structure asymmetric outside the boundary layer, much like boundary-layer separation in high-Reynolds-number flows transports vorticity throughout the fluid. Indeed, in figures 1 and 2 one can see that the asymmetric structure is present at $O(1)$ distances from particle contact. This transport throughout the bulk takes place in a time of order $1 / \dot{\gamma}$, which can be viewed either as the advective time or, perhaps more appropriately, as the diffusive time for the structure to develop on the length $b$ with the shear-induced diffusivity that scales hydrodynamically as $\dot{\gamma} b^{2}$ (cf. $\S 6$ ). The time scale for the boundary layer to develop is the diffusive time in the boundary layer $b^{2}\left(P e_{b}^{-1}\right)^{2} / D \sim\left(P e_{b}\right)^{-1} / \dot{\gamma}$. Thus, the boundary-layer very rapidly develops an asymmetric structure and then the slower process of advection or shear-induced diffusion transports this asymmetric structure throughout the bulk. This effect on the bulk in turn influences the boundary-layer structure through the matching condition, so that a self-consistent structure independent of the initial state is reached in a time of $O(1 / \dot{\gamma})$. Thus, (22) should only be viewed as an estimate of the stress at high volume fractions.

As a final remark, we note that although the stress contribution at high $P e$ is from interparticle forces, the suspension will not display measurable viscoelastic phenomena. When flow is stopped for example, the structure in the boundary layer relaxes rapidly in a time of $O\left(\mathrm{Pe}_{b}^{-1} / \dot{\gamma}\right)$, and thus the Weissenberg (or Deborah) number given by the ratio of the time for material relaxation to the time scale of the bulk flow scales as $P e_{b}^{-1}$; a vanishing Weissenberg number corresponds to no viscoelasticity. Since motion has ceased, advection within the bulk will not occur, and any asymmetry at $O(1)$ particle separations will remain essentially frozen, ultimately relaxing on the Brownian diffusion time $a^{2} / D$. If flow is restarted in the same direction, only the boundary-layer asymmetry needs to be recreated, which appears to be instantaneous on the flow time scale. If the flow direction is reversed, then a new (reversed) asymmetric structure on $O(1)$ length scales must be created, which takes the advective time $\dot{\gamma}^{-1}$. The predictions of this analysis are in agreement with the observations of Gadala-Maria \& Acrivos (1980), although it should be noted that the predicted boundary-layer thickness for the $25 \mu \mathrm{m}$ particles used is only $10 \AA$ and such continuum analyses may no longer apply. For all practical purposes, the only important time scale at high $P e_{b}$ is the inverse of the shear rate. We note that the experiments of Gadala-Maria \& Acrivos involved suspensions in which hydrodynamic interactions were important. The microstructure of hydrodynamically interacting suspensions is analysed in the following two sections and the above conclusions are unchanged.

\section{Interparticle forces, pair hydrodynamics}

\subsection{Pair-distribution function with hydrodynamics}

The relatively simple analysis without hydrodynamics showed that there is a large $O(P e)$ excess of particle pairs along the compressional axes, leading to non-Newtonian rheology in the limit $P e \rightarrow \infty$. We now include hydrodynamic interactions and show that a similar microstructure is found for all non-zero $b-a$. The solution depends on $b$ from the evaluation of the hydrodynamic functions at $r=2 b$ used in the boundary-layer equation, and thus we are able to determine the manner in which the rheology approaches the pure-hydrodynamic limit of $b / a-1 \rightarrow 0$. Since we wish to consider all $b>a$, we scale all lengths with $a$, applying this scaling also to $b$ and 
denoting the dimensionless effective radius $\hat{b} \equiv b / a$. The Péclet number is now based on the length $a, P e=\dot{\gamma} a^{2} / 2 D$.

When hydrodynamics are included, the outer solution for dilute $\phi$ is $p(r)$, and as in $\S 3$, we stretch the radial coordinate near contact as

$$
y=P e(r-2 \hat{b}) .
$$

Expanding the hydrodynamic functions to linear terms about their values at $r=2 \hat{b}$, we find the equation for $g$ in the stretched coordinates,

$$
\begin{gathered}
\left(1+\alpha_{1} P e^{-1} y\right) \frac{\partial^{2} g}{\partial y^{2}}-\alpha_{2} \gamma_{r}\left(1-\frac{\alpha_{3}}{\gamma_{r}} P e^{-1}+\alpha_{4} P e^{-1} y\right) \frac{\partial g}{\partial y} \\
=\alpha_{5} P e^{-1} \gamma_{r} g+P e^{-1} \frac{(1-\bar{B})}{2 \bar{G}}\left[\gamma_{\theta} \frac{\partial g}{\partial \theta}+\frac{\gamma_{\varphi}}{\sin \theta} \frac{\partial g}{\partial \varphi}\right]+O\left(P e^{-2}\right), \\
\frac{\partial g}{\partial y}=\alpha_{2} \gamma_{r} g \quad \text { at } \quad r=2 \hat{b} \\
g \sim \bar{p} \quad \text { as } \quad y \rightarrow \infty,
\end{gathered}
$$

where

$$
\bar{p}=p(2 \hat{b})
$$

In (23a) and (23b), $\alpha_{1}-\alpha_{5}$ are given by

$$
\left.\begin{array}{c}
\alpha_{1}=\frac{\bar{G}^{\prime}}{\bar{G}}, \quad \alpha_{2}=\frac{2 \hat{b}(1-\bar{A})}{\bar{G}}, \quad \alpha_{3}=\frac{\bar{G}^{\prime}+\bar{G} / \hat{b}}{2 \hat{b}(1-\bar{A})} \\
\alpha_{4}=\frac{1-\bar{A}-2 \hat{b} \bar{A}^{\prime}}{2 \hat{b}(1-\bar{A})}, \quad \alpha_{5}=\frac{\bar{W}}{\bar{G}},
\end{array}\right\}
$$

where overbars are used to denote the hydrodynamic functions evaluated at $r=2 \hat{b}$, e.g. $\bar{G}=G(2 \hat{b})$. Note that the $\alpha_{i}$ are positive and independent of $P e$. The hydrodynamic functions $G(r)$ and $W(r)$ give the relative radial mobility of two particles and the divergence of the relative velocity, respectively (Batchelor 1977).

We divide $(23 a)$ by $\left(1+\alpha_{1} P e^{-1} y\right)$, discarding terms of $O\left(P e^{-2}\right)$ and the entire right-hand side to yield the radial balance

$$
\frac{\partial^{2} g}{\partial y^{2}}-\alpha_{2} \gamma_{r}\left[1-\frac{\alpha_{3}}{\gamma_{r}} P e^{-1}+\left(\alpha_{4}-\alpha_{1}\right) P e^{-1} y\right] \frac{\partial g}{\partial y}=0 .
$$

As in the problem without hydrodynamics, a solution satisfying the leading-order balance and the boundary conditions does not exist, making it necessary to retain the $O\left(P e^{-1}\right)$ terms in (25) to obtain a solution. The solution is

$$
g=\bar{p}\left[\frac{1+\alpha_{2} \gamma_{r} \int_{0}^{y} \mathrm{e}^{S(z)} \mathrm{d} z}{1+\alpha_{2} \gamma_{r} \int_{0}^{\infty} \mathrm{e}^{S(z)} \mathrm{dz}}\right], \quad \gamma_{r}<0,
$$

where

$$
S(z)=\alpha_{2} \gamma_{r}\left[\left(1-\frac{\alpha_{3}}{\gamma_{r}} P e^{-1}\right) z+\frac{\left(\alpha_{4}-\alpha_{1}\right)}{2} P e^{-1} z^{2}\right],
$$


and

$$
g(0)=-\alpha^{*} \gamma_{r} \bar{p} P e \quad \text { as } \quad P e \rightarrow \infty,
$$

with

$$
\alpha^{*}(b / a)=\frac{\alpha_{2}}{\alpha_{4}+\alpha_{2} \alpha_{3}-\alpha_{1}} .
$$

Again, the deviation of the pair-distribution function from the spherically symmetric $p$ is $O(P e)$ in the compressional quadrants, and there is no solution to (25) for $\gamma_{r}>0$.

As in the case without hydrodynamics, the $O\left(P^{-1}\right)$ terms neglected, including the velocity divergence term, $\alpha_{5} P e^{-1} g$, affect the precise value of $g$ at contact, but do not affect the scaling with $P e$.

\subsection{Macroscopic stress with hydrodynamics}

The particle stress is evaluated using (9)-(11). With hydrodynamics included, there are several contributions of $O(\eta \dot{\gamma})$ as $P e \rightarrow \infty$. First, we note that since $\nabla \cdot\left(\boldsymbol{R}_{S U} \cdot \boldsymbol{R}_{F U}^{-1}\right)$ is $O(1)$ everywhere and the product of $g(\boldsymbol{r})$ and the boundary-layer volume is also $O(1)$, the Brownian stress $(10 a)$ remains $O\left(k T / a^{3}\right)$, in agreement with the simulations of Phung et al. (1996) and Phung (1993). Both the hydrodynamic and interparticle-force stress contributions are $O(\eta \dot{\gamma})$, however. For the hydrodynamic stress this is obvious, while for the interparticle-force contribution we note that the hard-sphere force is $\boldsymbol{F}^{P}=\frac{1}{2} k T \hat{\boldsymbol{r}} \delta(r-2 b)$, with the $\frac{1}{2} k T$ amplitude necessary to give the correct equilibrium osmotic pressure. Hence, in dimensional form

$$
n\left\langle\boldsymbol{S}^{P}\right\rangle=-n^{2} k T \oint_{r=2 b}\left(b \hat{\boldsymbol{r}} \hat{\boldsymbol{r}}+\boldsymbol{R}_{S U} \cdot \boldsymbol{R}_{F U}^{-1} \cdot \hat{\boldsymbol{r}}\right) g(\boldsymbol{r}) \mathrm{d} S,
$$

and because $g(2 b)$ is $O(P e)$, this 'contact' integral will give an $O(\eta \dot{\gamma})$ contribution to the stress. Making use of the known hydrodynamic functions, (28) can be rewritten as

$$
n\left\langle\mathbf{S}^{P}\right\rangle=-n^{2} k T b(1-\bar{A}) \oint_{r=2 b} \hat{r} \hat{r} g(\boldsymbol{r}) \mathrm{d} S .
$$

Using (26) for $g(0)$, the interparticle-force contribution to the stress becomes

$$
n\left\langle\boldsymbol{S}^{P}\right\rangle=\eta \dot{\gamma} \phi^{2}\left(\frac{b}{a}\right)^{3}(1-\bar{A}) \alpha^{*} \bar{p} \frac{27}{4 \pi} \int_{\gamma_{r}<0} \hat{\boldsymbol{r}} \hat{\boldsymbol{r}} \gamma_{r} \mathrm{~d} \Omega .
$$

Note that the dependence on flow type is the same as in the absence of hydrodynamics; only the coefficient is modified by hydrodynamics.

In the limit of large $b / a$, the interparticle-force stress reduces to (19), the result found in the absence of hydrodynamics. For $b / a$ near unity we can estimate the limiting behaviour by noting that

$$
(1-\bar{A}) \sim(b / a-1), \alpha^{*} \sim(b / a-1), \quad \text { and } \quad \bar{p} \sim(b / a-1)^{-0.78} \quad \text { as } \quad b / a \rightarrow 1,
$$

so that

$$
n\left\langle\boldsymbol{S}^{P}\right\rangle \sim \eta \dot{\gamma} \phi^{2}(b / a-1)^{1.22} \int_{\gamma_{r}<0} \hat{\boldsymbol{r}} \hat{\boldsymbol{r}} \gamma_{r} \mathrm{~d} \Omega \quad \text { as } \quad b / a \rightarrow 1 .
$$

Thus, the interparticle force contribution to the stress vanishes with an exponent of 1.22 as the pure hydrodynamic limit is approached.

Unlike $n\left\langle\boldsymbol{S}^{P}\right\rangle$, where the integration is restricted to the boundary layer on the compressive axes, the hydrodynamic stress $n\left\langle\boldsymbol{S}^{H}\right\rangle$ is always $O(\eta \dot{\gamma})$ and contributions 
arise from the whole domain $r>2 b$. Thus, we cannot make general statements about the form of the hydrodynamic stress with regard to the asymmetry caused by the boundary layer because there is presumably an $O(1)$ angular variation on the extensional axes that we have not determined. Nevertheless, we can illustrate the consequences of the interparticle forces in creating an asymmetric structure by computing the second normal stress difference and viscosity for planar extensional flow from the compressional quadrant only. Batchelor \& Green (1972b) showed that for $b \equiv a$, the hydrodynamic stress associated with the spherically symmetric $p(r)$ is Newtonian; despite the divergence of $p$ at $r=2 a$, the viscosity is finite and the symmetry of the microstructure leads to zero normal stress differences. by

The second normal stress difference from the hydrodynamic stresslet, $N_{2}^{H}$, is given

$$
N_{2}^{H}=\left\langle\Sigma_{22}^{H}-\Sigma_{33}^{H}\right\rangle=\eta \dot{\gamma} \phi^{2} \frac{15}{4 \pi} I_{2}(b / a, P e),
$$

where the dimensionless integral $I_{2}$ is given by

$$
I_{2}(b / a, P e)=\int\left(\hat{S}_{22}^{H}-\hat{S}_{33}^{H}\right) g(\boldsymbol{\rho}) \mathrm{d} \boldsymbol{\rho},
$$

and $\boldsymbol{\rho}=\boldsymbol{r} / \boldsymbol{a}$ is used to emphasize the non-dimensionality. The hydrodynamic stresslet has been made non-dimensional by the isolated-particle value $20 \pi \eta a^{3} \dot{\gamma} / 3$. In figure $3(a), I_{2}(1.025, P e)$ is plotted, showing that it becomes independent of $P e$ as $P e \rightarrow \infty$, with an asymptotic value of the stress difference reached when $P e \approx 10^{3}$. Variation of $N_{2}^{H}$ with $b / a$ is demonstrated in figure 4 by plotting $I_{2}\left(b / a, 10^{6}\right)$ against $b / a-1$. For $b / a-1 \rightarrow 0, N_{2}^{H}$ vanishes as $(b / a-1)^{0.22}$ because $g$ is proportional to $(b / a-1)^{0.22}$. This slow decay as $b \rightarrow a$ suggests that even for very short-ranged forces, the normal stress differences of a non-colloidal suspension should be measurable.

At large $b / a$, the predominant hydrodynamic particle stress is given by the Einstein viscosity correction $(5 \phi / 2) \eta \boldsymbol{E}$. Interestingly, the two stress differences $N_{2}^{P}$ and $N_{2}^{H}$ scale differently as $b / a \rightarrow \infty: N_{2}^{H} \sim \eta \dot{\gamma} \phi^{2}$ and $N_{2}^{P} \sim \eta \dot{\gamma} \phi^{2}(b / a)^{5}$. The lack of dependence of $N_{2}^{H}$ upon $b / a$ is surprising, but results simply from the fact that the boundary-layer volume is proportional to $b^{3}$ while the stress experienced by one particle due to the straining field of a second scales as $(a / b)^{3}$.

While, as noted, general statements about the viscosity cannot be made because of the possible $O(1)$ variation of $g(\boldsymbol{r})$ along the extensional axes, it is nonetheless of interest to evaluate the influence of the boundary-layer structure of $g(\boldsymbol{r})$ upon the hydrodynamic contribution to the viscosity. The bulk hydrodynamic stress due to pair interactions is given in terms of the average stresslet experienced by a particle as

$$
\begin{aligned}
n\left(\left\langle\boldsymbol{S}^{H}\right\rangle-\boldsymbol{S}_{0}\right) & =n^{2} \int\left(\left\langle\boldsymbol{S}^{H}(\boldsymbol{r})\right\rangle_{2}-\boldsymbol{S}_{0}\right) g(\boldsymbol{r}) \mathrm{d} \boldsymbol{r} \\
& =n^{2} \int\left(\left\langle\boldsymbol{S}^{H}(\boldsymbol{r})\right\rangle_{2}-\boldsymbol{S}_{0}\right) p(r) \mathrm{d} \boldsymbol{r}+n^{2} \int\left(\left\langle\boldsymbol{S}^{H}(\boldsymbol{r})\right\rangle_{2}-\boldsymbol{S}_{0}\right)[g(\boldsymbol{r})-p(r)] \mathrm{d} \boldsymbol{r},
\end{aligned}
$$

where $\boldsymbol{S}_{0}=20 \pi \eta a^{3}\langle\boldsymbol{E}\rangle / 3$ is the stresslet of an isolated sphere and gives the Einstein viscosity correction. The first integral in the second line of (30) would yield the dilute-limit $6.95 \phi^{2} \eta$ contribution to the viscosity determined by Batchelor \& Green $(1972 b)$ if the full range of integration $r>2 a$ were allowed. Since $r$ is restricted to $r>2 b$, the coefficient depends on $b / a$ and is reduced from its pure-hydrodynamic value. For example, at $b / a=1.025$ the coefficient is 5.1. 

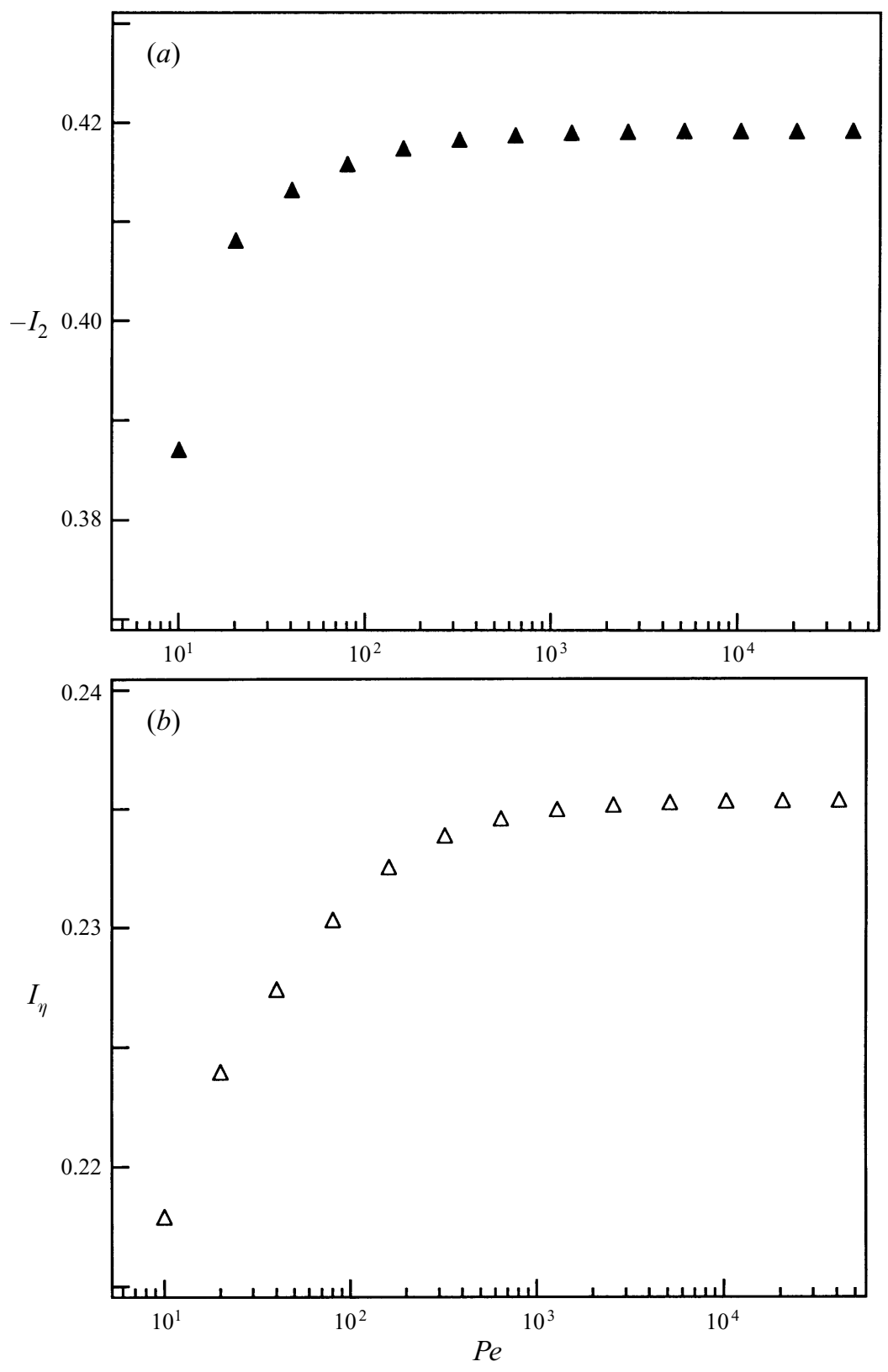

FIGURE 3. The dimensionless integrals $(a) I_{2}$ and $(b) I_{\eta}$ specifying the hydrodynamic second normal stress difference, $N_{2}^{H}=(15 / 4 \pi) \eta \dot{\gamma} \phi^{2} I_{2}$, and viscosity, $\eta^{+}=(15 / 4 \pi) \eta \phi^{2} I_{\eta}$, contributions as a function of $P e$ for $b / a=1.025$.

The boundary-layer contribution to the stress is given by the final integral of (30). In the dilute limit, the hydrodynamic contribution to the shear stress is conveniently expressed in terms of an additional $O\left(\phi^{2}\right)$ viscosity which we denote as $\eta^{+}$:

$$
\eta^{+} \dot{\gamma}=n^{2} \int\left(\left\langle S_{12}^{H}(\boldsymbol{r})\right\rangle_{2}-S_{0,12}\right)[g(\boldsymbol{r})-p(r)] \mathrm{d} \boldsymbol{r} .
$$




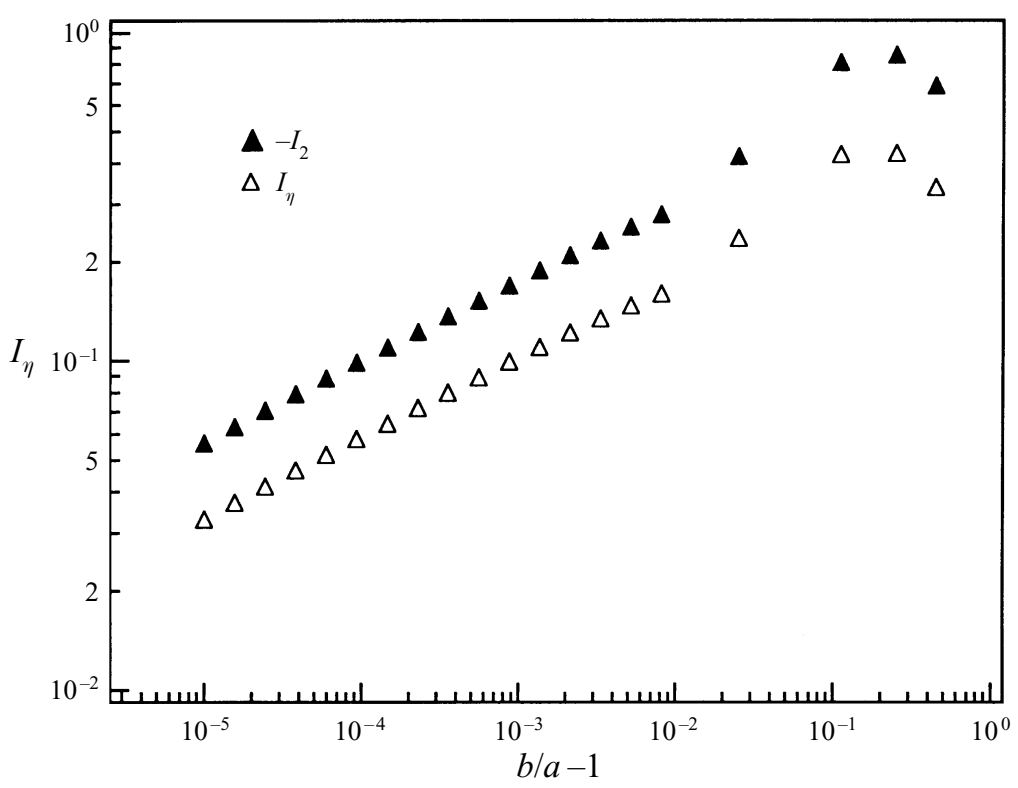

FIGURE 4. The dimensionless integrals $I_{2}$ and $I_{\eta}$ specifying the hydrodynamic second normal stress difference, $N_{2}^{H}=(15 / 4 \pi) \eta \dot{\gamma} \phi^{2} I_{2}$, and viscosity, $\eta^{+}=(15 / 4 \pi) \eta \phi^{2} I_{\eta}$, contributions as a function of $b / a-1$ for $P e=10^{6}$.

Using $g$ as given by (26), and scaling the stresslet as before with the isolated particle value, we write

$$
\eta^{+}=\frac{15}{4 \pi} \phi^{2} \eta I_{\eta}
$$

where

$$
I_{\eta}(b / a, P e)=\int\left(\hat{S}_{12}^{H}(\boldsymbol{\rho})-\hat{S}_{0,12}\right)[g(\boldsymbol{\rho})-p(\rho)] \mathrm{d} \boldsymbol{\rho} .
$$

Evaluation of $I_{\eta}$ for $b / a=1.025$ shows that $\eta^{+}$grows with $P e$ as illustrated in figure $3(b)$, reaching an asymptotic value at $P e \approx 10^{4}$. Hence, the hydrodynamic boundary-layer contribution to the viscosity is shear thickening. In figure 4, we see that $\eta^{+}$approaches zero in the same fashion as $N_{2}^{H}$ as $b \rightarrow a$. Recent unpublished numerical calculations of Brady \& Vicic (1997) have shown that with the inclusion of hydrodynamics the suspension viscosity in simple-shear flow shear thins at low Péclet number owing to the loss of the Brownian contribution to the stress, and then shear thickens at high Péclet number owing to this boundary-layer phenomenon.

In figure 4 the magnitudes of $I_{2}$ and $I_{\eta}$ are seen to go through maxima larger than the value obtained by extending their asymptotes for $b / a-1 \rightarrow 0$. This results from the fact that $p(r)$ varies less rapidly as $r$ increases and thus $I_{2}$ or $I_{\eta}$ are underpredicted by the form used in obtaining the asymptotic results. Note that the slope with which $I_{2}$ approaches zero differs by about $10 \%$ from 0.22 due to the influence of the logarithmic factor in $p(r)$ (cf. (40)).

\subsection{Extension to higher concentrations}

Because the origin of the asymmetry comes from the residual effects of Brownian motion in the boundary layer, we can extend these results to higher concentration by considering two factors. First, as discussed in $\$ 3.3$, the pair-distribution function 
asymptotes to $g^{\infty}(2 \hat{b} ; \phi)$ rather than to 1 outside the boundary layer, and the discussion in $\$ 3.3$ applies, although now $g^{\infty}(2 \hat{b} ; \phi)$ must be determined self-consistently including hydrodynamic interactions. Note also, that because the hydrodynamic contribution to the stress occurs throughout the entire volume $r>\hat{b}$, the behaviour of $g^{\infty}(\boldsymbol{r} ; \phi)$ for all $\boldsymbol{r}$ is needed; the relative importance of the contributions from these two regions is not known, however, but it is likely that the boundary-layer contribution dominates.

The second effect we must consider is a rescaling of the Péclet number when hydrodynamics are included. The boundary layer results from a balance between Brownian diffusion, $\boldsymbol{D} \cdot \nabla g$, and advection, $\boldsymbol{U g}$, and thus the relevant Péclet number should be $\bar{P} e=\left(\dot{\gamma} a^{2} / 2 D\right) \times|\hat{\boldsymbol{U}}| /|\hat{\boldsymbol{D}}|$, where $\hat{\boldsymbol{U}}$ and $\hat{\boldsymbol{D}}$ are the nondimensional relative velocity and diffusivity, respectively, and || signifies the magnitude. We now wish to argue that the ratio $|\hat{\boldsymbol{U}}| /|\hat{\boldsymbol{D}}|$ scales roughly as $1 / \hat{D}_{0}^{s}(\phi)$, where $\hat{D}_{0}^{s}(\phi)$ is the nondimensional short-time self-diffusivity, or just the relative mobility, at the volume fraction and flow conditions of interest, and thus the appropriate Péclet number is

$$
\overline{P e}=\frac{\dot{\gamma} a^{2}}{2 D_{0}^{s}(\phi)} .
$$

Although we need to examine the behaviour near contact, let us first consider the average relative motion of two widely separated particles. In this case, we know that the relative diffusivity asymptotes to $2 D_{0}^{s}(\phi)$, rather than to the infinite dilution value $2 D$; a particle's short-time mobility is hindered due to hydrodynamic interactions with its neighbours. We also know that the relative velocity of the pair is still that of the imposed linear shear flow $\dot{\boldsymbol{\Gamma}} \cdot \boldsymbol{r}$. Thus, at large distances from contact, the appropriate Péclet number is $\overline{P e}$. In addition to affecting a particle's mobility, hydrodynamic interactions also increase the shearing force exerted on a particle: since a particle's mobility has been reduced, the shearing force must increase by the same factor so that the relative velocity remains $\dot{\boldsymbol{\Gamma}} \cdot \boldsymbol{r}$. One can view this increased shear force as the imposed shear rate acting through the 'effective viscosity' of the suspension. This effective viscosity is the high-frequency dynamic viscosity for the given microstructure. Indeed, the available experimental and theoretical data (at least for equilibrium microstructures, Brady 1994) shows that the high-frequency dynamic viscosity is inversely proportional to the short-time self-diffusivity.

Now, as two particles approach contact, their relative motion is resisted by the solvent (as opposed to the suspension) viscosity, and thus the relative mobility vanishes linearly (for $b / a-1 \ll 1)$ as $(r-2)$ with the same slope as if the two particles were alone in the fluid; the lubrication behaviour of $\boldsymbol{D}$ is unaffected by concentration. The relative mobility measures the response to a unit force, and since only the solvent can exist between two nearly touching particles, the relative mobility remains the same. Thus at high concentration, the relative mobility should vanish at contact in the same manner as at infinite dilution and asymptote to the short-time self-diffusivity at large distances. The Stokesian Dynamics simulations of a monolayer at areal fraction 0.45 reported by Bossis, Brady \& Mathis (1988) show precisely this behaviour.

As particles approach one another, the relative velocity due to shearing flow also vanishes linearly with surface separation as the lubrication interactions require, but now with a concentration-dependent slope that is enhanced over its infinite dilution value. The reason for this altered slope is that, while the relative mobility is the same as at infinite dilution, the hydrodynamic shearing force is enhanced by the effective viscosity, just as was the case when the particles were widely separated. Particles are 
being driven together by the high-frequency dynamic viscosity, but the resistance to this motion is by the fluid viscosity. This enhancement of the slope of the relative velocity near contact was observed in the simulations of Bossis et al. mentioned above and in the simulations of Brady \& Bossis (1985) at an areal fraction of 0.4. Indeed, the results of Bossis et al. show that the ratio $\left(\langle\boldsymbol{U} \cdot \hat{\boldsymbol{r}}\rangle_{2} /\langle\boldsymbol{D} \cdot \hat{\boldsymbol{r}}\rangle_{2}\right) /(\boldsymbol{U} \cdot \hat{\boldsymbol{r}} / \boldsymbol{D} \cdot \hat{\boldsymbol{r}})$ is, to a good approximation, independent of $r$. Here, \langle\rangle$_{2}$ denotes the conditional average with two particles fixed in the concentrated suspension, while $\boldsymbol{U}$ is the relative velocity of two particles alone in the fluid.

Thus, we take as an estimate that near contact

$$
|\hat{\boldsymbol{U}}| /|\hat{\boldsymbol{D}}| \approx D / D_{0}^{s}(\phi),
$$

and that the rescaled Péclet number should be as given in (32). Alternatively, noting that $1 / D_{0}^{s}(\phi) \approx 6 \pi \eta_{\infty}^{\prime}(\phi) a / k T$, where $\eta_{\infty}^{\prime}(\phi)$ is the high-frequency dynamic viscosity, reflecting the fact that the shearing force is enhanced, the appropriate Péclet number can also be written as

$$
\overline{P e}=\frac{\dot{\gamma} a^{2}}{2 D_{0}^{s}(\phi)}=\frac{3 \pi \eta_{\infty}^{\prime}(\phi) \dot{\gamma} a^{3}}{k T} .
$$

Clearly, a complete determination of the conditionally averaged hydrodynamic interactions between two particles in a concentrated suspension is needed in order to substantiate this scaling estimate. It is worth noting that Brady (1993b) showed that (32) was the appropriate rescaled Péclet number for near-equilibrium suspensions, $\overline{\mathrm{Pe}} \rightarrow 0$, and now we see that it also appears to be the appropriate Péclet number as $\overline{P e} \rightarrow \infty$. It is natural to suppose, therefore, that $\overline{P e}$ is the appropriate rescaled Péclet for all Péclet numbers, and such a rescaling can be used to collapse viscosity data for all shear rates (Brady \& Vicic 1997, unpublished).

With this rescaled Péclet number, the hydrodynamic contribution to the stress from the boundary layer, which is dominant because of the additional factor of $(b / a-1)$ in the interparticle-force contribution, should scale as

$$
\left|n\left\langle\mathbf{S}^{H}\right\rangle\right|_{b l} \sim \eta \dot{\gamma}\left[(b / a-1)^{0.22} \phi^{2} g^{\infty}(2 \hat{b} ; \phi) / \hat{D}_{0}^{s}(\phi)\right] \times \text { an } O(1) \text { function of } \phi,
$$

at high concentrations. Both $g^{\infty}(2 \hat{b} ; \phi)$ and $1 / \hat{D}_{0}^{s}(\phi)$ should diverge as maximum packing is approached. For equilibrium suspensions we know both diverge as $(1-$ $\left.\phi / \phi_{m}\right)^{-1}$ as $\phi \rightarrow \phi_{m}$, with $\phi_{m} \approx 0.64$, but at what volume fraction and with what exponent the divergence occurs at infinite Péclet number is unknown. The scaling in (33) can be interpreted physically as follows: there are $n$ particles per unit volume each contributing an average stresslet that is proportional to $(b / a-1)^{0.22} \phi g^{\infty}(2 \hat{b} ; \phi)$, from the number of contacting pairs in the boundary layer, and to $\eta \dot{\gamma} a^{3} / \hat{D}_{0}^{s}(\phi)$, from the stress due to the enhanced shearing force $\eta \dot{\gamma} a^{2} / \hat{D}_{0}^{s}(\phi)$.

The scaling of the boundary-layer stress in (33) has a number of important implications. First, we note the residual dependence on the interparticle forces through $(b / a-1)^{0.22}$. Thus, effects such as interparticle forces and small-scale surface roughness should be manifest in viscosity measurements (as well as in normal stress differences) at high Péclet number. This may explain why the experimental data at high concentrations for non-Brownian suspensions do not collapse onto a single curve (the variation at a given volume fraction is an order of magnitude), even though the measurements of an individual researcher are very reproducible (Thomas 1965). Second, (33) suggests that the divergence in the stress with volume fraction should be the same for all suspensions, which is also in keeping with correlations for non-Brownian suspensions, 
which show a divergence with an exponent near 2. This last point supposes that the contribution to the hydrodynamic stress from the volume outside the boundary layer is no more singular than the boundary-layer contribution, an assertion that needs to be tested.

The last issue we need to address is whether the exponent 0.22 of the dependence on $(b / a-1)$ is affected by the rescaled hydrodynamics in concentrated suspensions. The exponent 0.22 comes from the singular behaviour of the pure-hydrodynamic pair-distribution function $p(r)$ scaling as $(r-2)^{-0.78}$ near contact. As will become clear in the next section, the exponent on $p(r)$, which we shall call $\delta$, is given in terms of the contact values of hydrodynamic functions:

$$
\delta=\frac{1}{2} \frac{W(2)}{A^{\prime}(2)} \doteq-0.78 .
$$

Here, $W$ is the divergence of the relative velocity and is given by $W(r)=3(B-A)-r A^{\prime}$, where $A$ and $B$ give the relative radial and tangential velocity due to the imposed linear flow. Thus, $W$ should scale with the effective suspension viscosity in the same manner as the relative velocity, and to a first approximation $\delta$ should be unaffected by concentration. It would be interesting to see if this is borne out by the conditionally averaged hydrodynamic interactions.

Finally, it is worth noting that the relaxation time in the boundary layer now becomes $\left(a \bar{P} e^{-1}\right)^{2} / D(2 \hat{b}) \sim \dot{\gamma}^{-1} \bar{P} e^{-1} / \bar{G}$, scaling with $\bar{P} e$ and with $\bar{G}$, the relative mobility of two particles along their line of centres at the minimum separation $2 \hat{b}$; for $b / a \approx 1, \bar{G} \sim 2(b / a-1)$.

\section{Pure hydrodynamics}

We saw in the previous section that the effects of the boundary layer all vanish as the pure-hydrodynamic limit, $b \equiv a$, is approached. To understand why this occurs, and why the pure-hydrodynamic limit is a singular case within the singular problem as $P e \rightarrow \infty$, we consider the dilute limit of two particles in a pure straining motion. In pure straining motion there are no regions of closed streamlines and therefore the spherically symmetric $g(\boldsymbol{r})=p(r)$ applies at infinite $P e$. The vanishing of the relative velocity at contact results in a boundary layer on both the compressional and extensional sides. The divergence of the pure-hydrodynamic pair-distribution function as $p(r) \sim(r-2)^{-0.78}$, which comes from the hydrodynamic functions in (34), results in an $O\left(P e^{0.78}\right)$ buildup of particles in the boundary layer. Although this accumulation is large, its influence vanishes in the pure-hydrodynamic limit: the $O\left(P e^{0.78}\right)$ buildup occurs in the $O\left(a^{3} P e^{-1}\right)$ volume of the boundary layer and thus there is not an $O(1)$ asymmetry in microstructure as $P e \rightarrow \infty$. The suspension is therefore Newtonian, and approaches this limit as $P e^{-0.22}$.

The steady pair equation in spherical coordinates is

$$
\begin{aligned}
\frac{1}{r^{2}} \frac{\partial}{\partial r}\left[r^{2} G(r) \frac{\partial g}{\partial r}\right] & +\frac{H}{r^{2} \sin \theta}\left[\frac{\partial}{\partial \theta} \sin \theta \frac{\partial g}{\partial \theta}+\frac{1}{\sin \theta} \frac{\partial^{2} g}{\partial \varphi^{2}}\right] \\
& -P e\left[U_{r} \frac{\partial g}{\partial r}+\frac{U_{\theta}}{r} \frac{\partial g}{\partial \theta}+\frac{U_{\varphi}}{r \sin \theta} \frac{\partial g}{\partial \varphi}+g \nabla \cdot U\right]=0 .
\end{aligned}
$$

The components of the relative velocity are

$$
U_{r}=r[1-A(r)] \gamma_{r}, \quad U_{\theta}=r[1-B(r)] \gamma_{\theta}, \quad \text { and } \quad U_{\varphi}=r[1-B(r)] \gamma_{\varphi},
$$


and the divergence of $\boldsymbol{U}$ is

$$
\nabla \cdot \boldsymbol{U}=W(r) \gamma_{r}
$$

The hydrodynamic functions $G(r)$ and $H(r)$ give the relative diffusivity parallel and perpendicular to the line of centres, respectively.

In the boundary layer the radial coordinate is stretched according to

$$
y=\operatorname{Pe}(r-2),
$$

and the diffusivity and velocity are approximated by Taylor expansions to linear terms about their values at $r=2$. At leading order in $P e$ the governing equation for $g$ in the boundary layer and the accompanying boundary conditions are

$$
\begin{aligned}
& y \frac{\partial^{2} g}{\partial y^{2}}+\left[1+\gamma_{r} A^{\prime}(2) y\right] \frac{\partial g}{\partial y}- {\left[\frac{1}{2}(1-B(2))\left(\gamma_{\theta} \frac{\partial g}{\partial \theta}+\frac{\gamma_{\varphi}}{\sin \theta} \frac{\partial g}{\partial \varphi}\right)\right]+\frac{1}{2} W(2) \gamma_{r} g=0, } \\
& y \frac{\partial g}{\partial y}=0 \quad \text { at } \quad y=0, \\
& g \sim p \quad \text { as } \quad y \rightarrow \infty,
\end{aligned}
$$

where a prime denotes differentiation of a hydrodynamic function with respect to $r$, and we have used $A(2)=1, G(2)=0$, and $G^{\prime}(2)=2$. Values of other quantities appearing in $(35 a)$ are

$$
A^{\prime}(2)=-4.08, \quad B(2)=0.406, \quad \text { and } \quad W(2)=6.38 .
$$

The system of equations $(35 a)-(35 c)$ admits the similarity transformation

$$
g(y, \theta, \varphi)=u(\xi) h(\theta, \varphi), \quad \text { where } \quad \xi=y / Y(\theta, \varphi)
$$

and $u$ is governed by

$$
\begin{gathered}
\xi u^{\prime \prime}+(1+\alpha \xi) u^{\prime}-\delta u=0, \\
\xi u^{\prime}=0 \quad \text { at } \quad \xi=0, \\
u h \sim p(r) \quad \text { as } \quad \xi \rightarrow \infty .
\end{gathered}
$$

The equations governing $Y$ and $h$ are

$$
\gamma_{\theta} \frac{\partial Y}{\partial \theta}+\frac{\gamma_{\varphi}}{\sin \theta} \frac{\partial Y}{\partial \varphi}+\gamma_{r} \frac{4 A^{\prime}(2)}{1-B(2)} Y=\frac{4 \alpha}{1-B(2)},
$$

and

$$
\gamma_{\theta} \frac{\partial h}{\partial \theta}+\frac{\gamma_{\varphi}}{\sin \theta} \frac{\partial h}{\partial \varphi}+\gamma_{r} \frac{2 W(2)}{1-B(2)} h=\frac{4 \delta h}{Y(1-B(2))} .
$$

Without loss of generality† we set $\alpha=1$, while $\delta$ is determined by matching $u h$ to $p$, a condition which requires the angular dependence of $u h$ to vanish at large $\xi$. With $\alpha=1,(36 a)$ shows that

$$
u \sim \text { constant } \times \xi^{\delta} \quad \text { as } \quad \xi \rightarrow \infty .
$$

Near particle contact, Batchelor \& Green (1972b) determined

$$
p \sim 0.234(r-2)^{-0.78}\left[\log (r-2)^{-1}\right]^{-0.29} \text { as } \quad r \rightarrow 2,
$$

$\dagger$ A study of (37) shows that $\alpha$ must be positive, but is otherwise arbitrary, in order that $Y$ (and hence the boundary-layer thickness) be positive. At the stagnation points in the compressional quadrants, e.g. $\theta=\pi / 2$ and $\varphi=3 \pi / 4$, both $\gamma_{\theta}$ and $\gamma_{\varphi}$ vanish while $\gamma_{r}<0$. Thus, at the stagnation point $Y=\alpha / A^{\prime}(2) \gamma_{r}$, and because $A^{\prime}<0$ we must have $\alpha>0$. 
and up to a weak logarithmic error, matching of the radial dependence of $u$ and $p$ is accomplished by taking $\delta=-0.78$. This value of $\delta$ also satisfies the requirement of vanishing angular dependence of the product $u h$, as is verified by inserting $h=Y^{\delta}$ into (38), which yields an equation identical to (37) when we choose

$$
\delta=\frac{1}{2} \frac{W(2)}{A^{\prime}(2)} \doteq-0.78,
$$

previously given by (34), which shows the origin of the divergence in $p$ in terms of hydrodynamic functions.

The details of the similarity solution are given in the Appendix. The final form and $P e$-dependence of the pair-distribution function in the boundary layer are given by

$$
g=k(\theta, \varphi)\left[\frac{P e}{Y(\theta, \varphi)}\right]^{0.78} M(0.78,1,-y / Y(\theta, \varphi)),
$$

where $M$ is the confluent hypergeometric function. The weak logarithmic dependence of the outer solution $p$ is neglected in writing (41), but may be included in the angularly dependent coefficient $k(\theta, \varphi)$ if matching is performed. Matching to $p$ occurs at different $r$ for varying $\theta$ and $\varphi$, and thus the magnitude of $k$ will vary with angular position so that the logarithm may be absorbed.

The normal stress differences for purely hydrodynamically interacting hard spheres scale as the boundary-layer volume $O\left(a^{3} P e^{-1}\right)$ times $g(2)$ (cf. equation (11)), and thus non-Newtonian effects scale as $P e^{-0.22}$ as $P e \rightarrow \infty$. This slow decay suggests measurable normal stress differences may be expected even at large $P e$. Ultimately, however, as $P e \rightarrow \infty$ the structure becomes symmetric and the rheology Newtonian. Although we have carried out a detailed analysis for planar extensional flow only, we expect the same conclusion to hold for general linear flows.

The relatively weak $O\left(P e^{0.78}\right)$ buildup of particles in the boundary layer is directly related to the singular behaviour of $p(r)$ near contact. From the discussion in $\S 4.3$ concerning the behaviour of the exponent $\delta$ in (34), it seems unlikely that $\delta$ will decrease sufficiently (it needs to be -1 ) with increasing $\phi$ to result in an $O(1)$ asymmetry as $P e \rightarrow \infty$. The pure-hydrodynamic limit is expected to remain a special, singular, case.

It is also interesting to note that the relaxation time in the boundary layer now changes its scaling. Since the boundary layer exists all the way up to contact, the relative diffusivity in the boundary layer is proportional to the boundary layer thickness, and the relaxation time in the boundary layer now scales as $\left(a P e^{-1}\right)^{2} / D P e^{-1} \sim \dot{\gamma}^{-1}$, showing that the only time scale operative in the pure-hydrodynamic limit is the inverse shear rate.

\section{Shear-induced self-diffusivity of hard spheres}

A theory to describe the self-diffusivity of a sheared suspension at arbitrary $\phi, P e$, and lengthscale of the particle-fraction disturbance has been developed by Morris \& Brady (1996). The theory is based upon the experimental technique of dynamic light scattering (Berne \& Pecora 1976) and amounts to a study of the Fourier-transformed equation governing the configurational transition probability. This method has been extensively used in the evaluation of diffusion in quiescent colloidal dispersions (Jones \& Burfield 1982; Rallison \& Hinch 1986; Pusey 1991; Brady 1994). Here we apply the theory to the determination of the self-diffusivity of a sheared suspension for $P e \gg 1$, a phenomenon first noted by Eckstein, Bailey \& Shapiro (1977). 
We first outline those aspects of the theory necessary for the present calculation. The self-diffusivity is determined from the temporal evolution of the spatial correlation of particle positions. The self-intermediate scattering function, $F_{s}$, which represents the Fourier transform of the spatial autocorrelation function, is given by

$$
\begin{aligned}
F_{S} & =\left\langle\mathrm{e}^{\mathrm{i} k \cdot\left[x_{1}(t)-\boldsymbol{x}_{1}(0)\right]}\right\rangle \\
& =\iint \mathrm{e}^{\mathrm{i} \boldsymbol{k} \cdot\left[x_{1}(t)-x_{1}(0)\right]} P_{N}\left(\boldsymbol{x}^{N}(t) \mid \boldsymbol{x}^{N}(0)\right) P_{N}^{0}\left(\boldsymbol{x}^{N}(0)\right) d \boldsymbol{x}^{N}(t) \boldsymbol{x}^{N}(0),
\end{aligned}
$$

where $\boldsymbol{k}$ is the wavevector of the scattered light, $P_{N}$ here denotes the probability for the configuration to undergo the transition from $\boldsymbol{x}^{N}(0)$ to $\boldsymbol{x}^{N}(t)$, and $P_{N}^{0}$ is the probability distribution for the configuration at the initial time, which we take to be the steady distribution at the conditions of interest.

For arbitrary flow conditions, the self-diffusivity may be defined as the limit as $k \rightarrow 0$ of the $-k^{2}$ coefficient in the derivative

$$
\frac{\partial \ln F_{s}}{\partial t} \equiv\left(\ln F_{s}\right) .
$$

In the general case, $\left(\ln F_{s}\right)$ is given by

$$
\begin{aligned}
\left(\ln F_{s}\right)= & \boldsymbol{k} \cdot \dot{\boldsymbol{\Gamma}} \cdot \nabla_{k} \ln F_{s}+\mathrm{i} \boldsymbol{k} \cdot\left\langle\boldsymbol{U}_{1}\right\rangle^{0}-\boldsymbol{k} \cdot\left\langle\boldsymbol{D}_{11}\right\rangle^{0} \cdot \boldsymbol{k} \\
& -\boldsymbol{k} \cdot \int\left(\boldsymbol{D}_{11}-\left\langle\boldsymbol{D}_{11}\right\rangle^{0}\right) \cdot \boldsymbol{k} f_{N} P^{0} \mathrm{~d} \boldsymbol{r}^{N}+\mathrm{i} \boldsymbol{k} \cdot \int \boldsymbol{U}_{1}^{\prime} f_{N} P^{0} \mathrm{~d} \boldsymbol{r}^{N} \\
& -\mathrm{i} \boldsymbol{k} \cdot \int \sum_{\alpha=2}^{N}\left[\left(\boldsymbol{D}_{1 \alpha}-\boldsymbol{D}_{11}\right) \cdot \nabla_{\alpha} f_{N}+\left(\boldsymbol{D}_{1 \alpha}-\boldsymbol{D}_{11}\right) f_{N} \cdot \nabla_{\alpha}\left(\ln P^{0}+V\right)\right] P^{0} \mathrm{~d} \boldsymbol{r}^{N},
\end{aligned}
$$

where $\boldsymbol{r}^{N}$ is the vector giving the positions of the other particles relative to particle 1 , which lies at the origin, and $P^{0}=P_{N-1 \mid 1}^{0}$ is the probability for the relative initial configuration. Also, $\left\langle\boldsymbol{U}_{1}\right\rangle^{0}$ is the average velocity of the reference particle, and

$$
\boldsymbol{U}_{1}^{\prime}=\boldsymbol{U}_{1}-\dot{\boldsymbol{\Gamma}} \cdot \boldsymbol{x}_{1}-\left\langle\boldsymbol{U}_{1}\right\rangle^{0}
$$

is the hydrodynamic disturbance velocity relative to the bulk flow (uniform plus shear) of the tagged particle. The scalar function $f_{N}$, which describes the perturbation to the microstructure caused by the motion of a tagged particle, is central to the theory of the long-time self-diffusivity because the difference between the short-time self-diffusivity, $k T\left\langle\boldsymbol{M}_{11}\right\rangle$, and the long-time self-diffusivity is due to the correlation of the flux of the tagged particle with $f_{N}$.

In the general case, $f_{N}$ is governed by a complex integro-differential equation (see equation (26) of Morris \& Brady 1996), which we do not reproduce here. For the long-time self-diffusivity, the steady $f_{N}$ for small $\boldsymbol{k}$ is sufficient, allowing considerable simplification:

$$
\begin{gathered}
\operatorname{Peg}\left\langle\boldsymbol{U} \cdot \nabla f_{N}\right\rangle_{2}^{0}-g\left\langle\boldsymbol{D} \cdot \nabla \bar{V} \cdot \nabla f_{N}\right\rangle_{2}^{0}-\nabla \cdot g\left\langle\boldsymbol{D} \cdot \nabla f_{N}\right\rangle_{2}^{0}-\nabla \cdot g \int P_{3 \mid 2}^{0}\left\langle\boldsymbol{D}_{23} \cdot f_{N}\right\rangle_{3}^{0} \mathrm{~d} \boldsymbol{r}_{3} \\
=\frac{1}{2} \mathrm{i} \boldsymbol{k} \cdot\left\{-P e g\left\langle\boldsymbol{U}^{\prime}\right\rangle_{2}^{0}+\nabla \cdot g\langle\boldsymbol{D}\rangle_{2}^{0}+g\langle\boldsymbol{D} \cdot \nabla \bar{V}\rangle_{2}^{0}\right\}+o(k),
\end{gathered}
$$

where \langle\rangle$_{2}^{0}$ stands for the conditional average over the initial distribution with two particles fixed, $P_{3 \mid 2}^{0}$ is the conditional probability for finding a particle at $\boldsymbol{r}_{3}$ given two 
particles fixed, and $\bar{V}=\ln P_{N}^{0}+V$. The boundary condition at contact is

$$
\begin{array}{r}
\hat{\boldsymbol{r}} \cdot\left[\left\langle\boldsymbol{D} \cdot \nabla f_{N}\right\rangle_{2}^{0}+\left\langle[\boldsymbol{D} \cdot \nabla \bar{V}] f_{N}\right\rangle_{2}^{0}+\int\left\langle\boldsymbol{D}_{23} \cdot \nabla_{3} f_{N}\right\rangle_{3}^{0} P_{3 \mid 2}^{0} d \boldsymbol{r}_{3}-P e\left\langle\boldsymbol{U} f_{N}\right\rangle_{2}^{0}\right] \\
=-\frac{1}{2} \hat{\boldsymbol{r}} \cdot\langle\boldsymbol{D}\rangle_{2}^{0} \cdot \mathrm{i} \boldsymbol{k} \text { at } \quad r=2,
\end{array}
$$

and the perturbation decays at large distances.

\subsection{Self-diffusion: no hydrodynamics}

For a dilute suspension of hard spheres with no hydrodynamics, the general equation for $f_{N}$ is reduced to that governing the steady pair perturbation $f_{2}$. Recognizing the linearity of the problem in $\mathrm{i} \boldsymbol{k}$, we write $f_{2}$ as

$$
f_{2}(\boldsymbol{r})=\mathrm{i} \boldsymbol{k} \cdot \boldsymbol{d},
$$

thus defining a wavevector-independent 'displacement' field $\boldsymbol{d}$. The steady equation and boundary conditions for $\boldsymbol{d}$ are

$$
\begin{aligned}
\nabla^{2} \boldsymbol{d}-\left(P e_{b} \dot{\boldsymbol{\Gamma}} \cdot \boldsymbol{r}\right. & -2 \nabla \ln g) \cdot \nabla \boldsymbol{d}=-\nabla \ln g, \\
\hat{\boldsymbol{r}} \cdot \nabla \boldsymbol{d} & =-\frac{1}{2} \hat{\boldsymbol{r}} \text { at } \quad r=2, \\
\boldsymbol{d} & \sim 0 \quad \text { as } \quad r \rightarrow \infty .
\end{aligned}
$$

Here, all lengths have been made dimensionless with $b$. Using $\boldsymbol{d}$, the expression for the long-time self-diffusivity of hard spheres can be expressed simply as

$$
\boldsymbol{D}_{\infty}^{s}=D \boldsymbol{I}-D \phi_{b} \frac{3}{\pi} \oint_{r=2} \hat{\boldsymbol{r}} \boldsymbol{d} g \mathrm{~d} \Omega .
$$

Only the contact value of $\boldsymbol{d}$ is needed for the evaluation of the hard-sphere diffusivity.

The steady $g(\boldsymbol{r})$ for hard spheres at $P e_{b} \gg 1$ was determined in $\S 3$ by a boundarylayer analysis, and there is a similar boundary-layer problem for $\boldsymbol{d}$. Only the radial portion of $\boldsymbol{d}$ is forced in (43a) and we write in the radial-balance approximation

$$
\boldsymbol{d}=d(r) \hat{\boldsymbol{r}},
$$

where $d(y)$ is governed by

$$
\begin{gathered}
\frac{\partial^{2} d}{\partial y^{2}}-\left[2 \gamma_{r}\left(1-\frac{P e_{b}^{-1}}{2 \gamma_{r}}+\frac{1}{2} P e_{b}^{-1} y\right)-2 \frac{\partial \ln g}{\partial y}\right] \frac{\partial d}{\partial y}=-P e_{b}^{-1} \frac{\partial \ln g}{\partial y}, \\
\frac{\partial d}{\partial y}=-\frac{1}{2} P e_{b}^{-1} \quad \text { at } \quad y=0 \\
d \sim 0 \quad \text { as } \quad y \rightarrow \infty .
\end{gathered}
$$

and $y=P e_{b}(r-2)$. The solution to this system is

$$
d(y)=P e_{b}^{-1} \int_{y}^{\infty}\left[\frac{1}{2} \frac{g^{2}(0)}{g^{2}} \mathrm{e}^{s(z)}+\frac{\mathrm{e}^{s(z)}}{g^{2}} \int_{0}^{z} \mathrm{e}^{-s(x)} g^{2}(x) \frac{\mathrm{d} \ln g}{\mathrm{~d} x} \mathrm{~d} x\right] \mathrm{d} z,
$$

where we recall that $s(z)$ was defined previously by (16). For the determination of $\boldsymbol{D}_{\infty}^{s}$, we need only the contact value of $d$, which upon evaluation of the integrals yields

$$
d(0)=-\frac{1}{3}+O\left(P e_{b}^{-1}\right) .
$$

Hence, recalling that $g(0)=-\frac{4}{3} P e_{b} \gamma_{r}$, the long-time self-diffusivity is given in dimen- 
sional form by

$$
\boldsymbol{D}_{\infty}^{s}=D \boldsymbol{I}-\dot{\gamma} b^{2} \phi_{b} \frac{2}{3 \pi} \int_{\gamma_{r}<0} \hat{\boldsymbol{r}} \hat{\boldsymbol{r}} \gamma_{r} \mathrm{~d} \Omega .
$$

In simple-shear flow $U_{1}=\dot{\gamma} x_{2}$, we have as $P e_{b} \rightarrow \infty$

$$
\boldsymbol{D}_{11}=\boldsymbol{D}_{22}=2 \boldsymbol{D}_{33}=\frac{16}{45 \pi} \dot{\gamma} b^{2} \phi_{b} ;
$$

the self-diffusivity in the vorticity direction is half that in the velocity gradient direction.

A comparison of (44) and (19) shows that $\boldsymbol{D}_{\infty}^{s}$ may be expressed in terms of $\boldsymbol{\Sigma}^{\prime}$ as

$$
\boldsymbol{D}_{\infty}^{s}=-\frac{b^{2}}{\eta} \frac{b}{a} \frac{1}{\phi_{b}} \frac{2}{27} \Sigma^{\prime}=-\frac{b^{2}}{\eta} \frac{b}{a} \frac{1}{27} \frac{\partial}{\partial \phi_{b}} \boldsymbol{\Sigma}^{\prime},
$$

a form that is of interest because it agrees with the idea of self-diffusion being driven by the osmotic, or partial, pressure: the self-diffusivity is the product of the particle mobility, which is 1 to $O(\phi)$, and the osmotic pressure gradient $\partial \boldsymbol{\Sigma}^{\prime} / \partial \phi$.

\subsection{Self-diffusion: pair hydrodynamic interactions}

With pair hydrodynamic interactions the governing equation for $\boldsymbol{d}$ in the boundary layer is

$$
\begin{gathered}
\frac{\partial^{2} \boldsymbol{d}}{\partial y^{2}}-\alpha_{2} \gamma_{r}\left[1-\frac{\alpha_{3}}{\gamma_{r}} P e^{-1}+\left(\alpha_{4}-\alpha_{1}\right) P e^{-1} y-2 \frac{\partial \ln g}{\partial y}\right] \frac{\partial \boldsymbol{d}}{\partial y}=\frac{1}{2} P e^{-1}\left(\frac{\overline{\boldsymbol{U}}_{r}^{\prime}}{\bar{G}}-2 \frac{\partial \ln g}{\partial y} \hat{\boldsymbol{r}}\right), \\
\frac{\partial \boldsymbol{d}}{\partial y}=-\frac{1}{2} P e^{-1} \hat{\boldsymbol{r}} \quad \text { at } y=0, \\
\boldsymbol{d} \sim 0 \quad \text { as } \quad y \rightarrow \infty,
\end{gathered}
$$

where we have neglected the angular derivatives and all lengths have been scaled by $a$. The velocity fluctuation $\overline{\boldsymbol{U}}^{\prime}$ is given by

$$
\overline{\boldsymbol{U}}^{\prime}=-2 \hat{b}[\hat{\boldsymbol{r}} \cdot\langle\boldsymbol{E}\rangle \cdot \hat{\boldsymbol{r}} \hat{\boldsymbol{r}} \bar{A}+\hat{\boldsymbol{r}} \cdot\langle\boldsymbol{E}\rangle \cdot(\boldsymbol{I}-\hat{\boldsymbol{r}} \hat{\boldsymbol{r}}) \bar{B}] .
$$

The expression for the long-time self-diffusivity now becomes

$$
\begin{aligned}
\boldsymbol{D}_{\infty}^{s}=D \boldsymbol{I} & -D \phi \hat{b}^{2} \bar{G} \frac{3}{\pi} \oint_{r=2 \hat{b}} \hat{\boldsymbol{r}} \boldsymbol{d} g \mathrm{~d} \Omega \\
& +D \phi \hat{b}^{2} \frac{3}{\pi} \int_{0}^{\infty} \oint_{r=2 \hat{b}}\left(P e^{-1}\left[\bar{G}^{\prime}+\frac{2}{r}(\bar{G}-\bar{H})\right] \hat{\boldsymbol{r}}-\frac{1}{2} \overline{\boldsymbol{U}}^{\prime}\right) \boldsymbol{d} g \mathrm{~d} \Omega \mathrm{d} y .
\end{aligned}
$$

The components of $\boldsymbol{d}$ in the radial balance approximation are

$$
\begin{aligned}
d_{r}(y) & =P e^{-1} \int_{y}^{\infty} \frac{\mathrm{e}^{S(z)}}{g^{2}(z)}\left[\frac{1}{2} g^{2}(0)+\int_{0}^{z} g^{2}(x) \mathrm{e}^{-S(x)}\left(-\frac{\bar{U}_{r}^{\prime}}{2 \bar{G}}+\frac{\mathrm{d} \ln g(x)}{\mathrm{d} x}\right) \mathrm{d} x\right] \mathrm{d} z, \\
d_{\theta, \varphi}(y) & =-P e^{-1} \frac{\bar{U}_{\theta, \varphi}^{\prime}}{2 \bar{G}} \int_{y}^{\infty} \frac{\mathrm{e}^{S(z)}}{g^{2}(z)} \int_{0}^{z} g^{2}(x) \mathrm{e}^{-S(x)} \mathrm{d} x \mathrm{~d} z .
\end{aligned}
$$

And their values at $y=0$ are, to leading order in $P e$,

$$
d_{r}(0)=P e^{-1} \frac{g(0)}{\bar{p}}\left[\frac{1}{2 \alpha_{2} \gamma_{r}}-\frac{\bar{U}_{r}^{\prime}}{2 \bar{G}} \frac{1}{\left(\alpha_{2} \gamma_{r}\right)^{2}}\right] \sim-\frac{\bar{A}}{2(1-\bar{A})} \frac{\alpha^{*}}{\alpha_{2}^{2}},
$$




$$
d_{\theta, \varphi}(0)=P e^{-1} \frac{\bar{U}_{\theta, \varphi}^{\prime}}{2 \bar{G}} \frac{g(0)}{\bar{p}} \frac{1}{\left(\alpha_{2} \gamma_{r}\right)^{2}} \sim-\frac{\gamma_{\theta, \varphi}}{\gamma_{r}} \frac{\bar{B}}{2(1-\bar{A})} \frac{\alpha^{*}}{\alpha_{2}^{2}},
$$

with $\alpha^{*}$ given by (27). The displacement field $\boldsymbol{d}$ is independent of both $P e$ and $(\hat{b}-1)$; the latter may be seen by noting that $1-\bar{A} \sim(\hat{b}-1)$ and $\alpha^{*} \sim(\hat{b}-1)$, while both $\bar{B}$ and $\alpha_{2}$ are $O(1)$.

Carrying out the volume integration with respect to $y$ in (46), noting that $\overline{\boldsymbol{U}}^{\prime}$ is constant to leading order in $P e$, and inserting $d(0)$, we have

$$
\begin{array}{r}
\boldsymbol{D}_{\infty}^{s}=\dot{\gamma} a^{2} \phi \bar{p} \hat{b}^{2} \frac{3}{4 \pi}\left(\frac{\alpha^{*}}{\alpha_{2}}\right)^{2}\left(-2 \hat{b}(1-\bar{A}) \oint_{\gamma_{r}<0} \hat{\boldsymbol{r}} \hat{\boldsymbol{r}} \gamma_{r} \mathrm{~d} \Omega+\oint_{\gamma_{r}<0} \hat{\boldsymbol{r}} \boldsymbol{U}^{\prime} \mathrm{d} \Omega\right. \\
\left.-\frac{1}{2 \bar{G} \alpha_{2}} \oint_{\gamma_{r}<0} \overline{\boldsymbol{U}}^{\prime} \overline{\boldsymbol{U}}^{\prime} \gamma_{r}^{-1} \mathrm{~d} \Omega\right)+O(D \ln P e) ;
\end{array}
$$

the $O(D \ln P e)$ error is from the volume integration. Of the three contributions to the long-time self-diffusivity in (49), the first two result from the first integral of (46), and scale respectively as $(\hat{b}-1)^{2.22}$ and $(\hat{b}-1)^{1.22}$. Comparing (49) with (44), we see that the first of these contributions yields the diffusivity in the absence of hydrodynamics. The third contribution to $\boldsymbol{D}_{\infty}^{s}$ scales as $(\hat{b}-1)^{0.22}$ and is therefore dominant for short-ranged forces. This final contribution has a form suggestive of the standard view of the self-diffusivity as $\boldsymbol{D}_{\infty}^{s} \sim \boldsymbol{U}^{\prime} \boldsymbol{U}^{\prime} \tau$, where $\tau$ is the time scale over which the velocity fluctuation is correlated. With this interpretation, $\tau$ is apparently proportional to $1 / \dot{\gamma} \gamma_{r}$.

We evaluate the long-time self-diffusivity predicted by (49) for the common example of simple-shear flow, $U_{1}=\dot{\gamma} x_{2}$. For this case, the final term of (49), containing the factor of $\gamma_{r}^{-1}$ in the integrand, results in divergent values of the 11 and 22 diffusivity components when applied directly. This can be explained by noting that the radial-balance approximation for $g$ (and hence for $\boldsymbol{d}$ ) is not valid for $\gamma_{r} \rightarrow 0$; in particular, the balance predicts that the asymmetric $g$ extends arbitrarily far from contact as $\gamma_{r}$ vanishes. While a solution for $g$ (and subsequently $\boldsymbol{d}$ ) including the angular terms would resolve the divergence, we simply cut off the integration slightly short of the divergent endpoint, appealing to the argument that, in (23a), the angular terms are comparable to the terms maintained in the radial-balance approximation for $\gamma_{r} \sim O\left(P e^{-1 / 2}\right)$. The corresponding physical justification for our ad hoc removal of the singular point is this: near $\gamma_{r} \rightarrow 0$, angular transport transports pairs efficiently and there is not a long residence time here (i.e. $\tau$ does not diverge) as predicted by the radial-balance approximation.

In figure 5, the long-time self-diffusivity components (scaled by $\dot{\gamma} a^{2} \phi$ ) for a dilute suspension in simple-shear flow are plotted. For ease of comparison with the results of a calculation of the self-diffusivity in a sheared suspension of rough spheres by da Cunha \& Hinch (1996), the diffusivity components are plotted against $2(b / a-1)$ rather than against $b / a-1$ as was the case for the stress components in figures 3 and 4. In figure $5(a), D_{22}$ is plotted, and we note that $D_{11}=D_{22}$; the two curves of figure 5(a) show results for two cut offs to remove the singularity in the $\varphi$ integration, the upper curve for $10^{-6} \pi$ removed from the $\varphi$ integration, and the lower with $10^{-4} \pi$ removed. Figure $5(b)$ displays $D_{33}$. In figure $5(c)$, we plot $-D_{12}$. This is the only non-zero 'off-diagonal' diffusivity component and is negative; the components $D_{i 3}$ and $D_{3 i}, i \neq 3$, are zero as may be seen from general symmetry considerations. 
Recently, da Cunha \& Hinch (1996) performed pair trajectory calculations to evaluate both the shear-enhanced self- and down-gradient diffusivities in a dilute sheared suspension of spheres with small-scale surface roughness. Particle roughness results in actual surface contact, thereby breaking the symmetry of the hydrodynamic trajectory of the pair much like the short-ranged repulsive force assumed in our study, and hence giving rise to a shear-enhanced self-diffusivity scaling as $\dot{\gamma} a^{2} \phi$ in the dilute limit. In the study of da Cunha \& Hinch, full pair hydrodynamics were employed, and the change in the displacement in the velocity gradient-vorticity plane over the entire pair interaction, and from this the diffusivity, was computed. The particle motion for the period of contact was determined for two models of the interaction at contact: in one model the particles rotated as a locked pair and in the other there was simultaneous rotation and slippage. Like the present work, da Cunha \& Hinch determined the shear enhancement of the diffusivity as a function of the minimum separation, and showed that it vanishes as $b-a \rightarrow 0$, using our notation. However, the manner in which $\boldsymbol{D}_{\infty}^{s}$ vanishes is apparently different from our result $\boldsymbol{D}_{\infty}^{s} \sim(b / a-1)^{0.22}$. Our results are only approximate because we used the radial-balance approximation for both $g$ and $\boldsymbol{d}$, while da Cunha $\&$ Hinch have studied the actual pair trajectory and therefore, in principle, have determined the correct diffusivity. Nevertheless, the magnitude of the predicted diffusivities in the vorticity direction, $D_{33}$, are very similar for short-ranged interparticle forces and roughness, and both studies find the diffusivity in the velocity gradient direction, $D_{22}$, to be much larger than $D_{33}$. Because $D_{12}$ was not considered in the trajectory analysis, we cannot make a comparison for this component.

\subsection{Extension to higher concentrations}

In order to estimate the scaling of the self-diffusivity at higher concentrations, the behaviour of $\boldsymbol{d}$ must be determined for large $\phi$. While many-particle effects undoubtedly have a quantitative influence on $\boldsymbol{d}$, it is possible to follow the same line of reasoning based on an effective pair interaction as we used in determining the stress scaling to estimate the $\phi$-dependence of $\boldsymbol{D}_{\infty}^{s}$.

We first note that the self-diffusivity is given by the correlated product of the velocity fluctuation $\times$ the displacement in an encounter; the $\boldsymbol{d}$-field is the displacement. If there were no singular boundary layer, the velocity fluctuation would integrate to zero owing to the symmetry of the infinite-Péclet-number encounter (actually $O\left(k T / \rho a^{3}\right)^{1 / 2}$ due to thermal effects).

Within the boundary layer the velocity fluctuation is $O(\dot{\gamma} a)$, the displacement during an encounter is $O(a)$, and the probability density of encounters $\times$ the boundary-layer volume is $O(\phi)$, yielding the $O\left(\phi \dot{\gamma} a^{2}\right)$ shear-induced self-diffusivity at low concentrations.

At higher concentrations in the absence of hydrodynamic interactions, the velocity fluctuation in the boundary layer remains $O(\dot{\gamma} a)$ - the 'velocity fluctuation' is due to the hard-sphere force arresting relative motion at the contact radius $b$. The displacement also remains $O(a)$, while the number of encounters is enhanced by the pair-distribution function at contact $g^{\infty}(2 ; \phi)$. That is, the only change in solving (43a) for $\boldsymbol{d}$ in the boundary layer is that $g$ must asymptote to $g^{\infty}(2 ; \phi)$ rather than to 1 . Thus, we expect $D_{\infty}^{s}(\phi) \sim \dot{\gamma} a^{2} \phi g^{\infty}(2 ; \phi)$ for all concentrations.

With hydrodynamic interactions, scaling all diffusivities with $2 D_{0}^{s}(\phi)$ in the expression (42) for $\boldsymbol{D}_{\infty}^{s}$ and the governing equation (43) for $\boldsymbol{d}$ results in the Péclet number $\overline{P e}$ used in the analysis of the stress at large $\phi$, and the boundary-layer thickness 

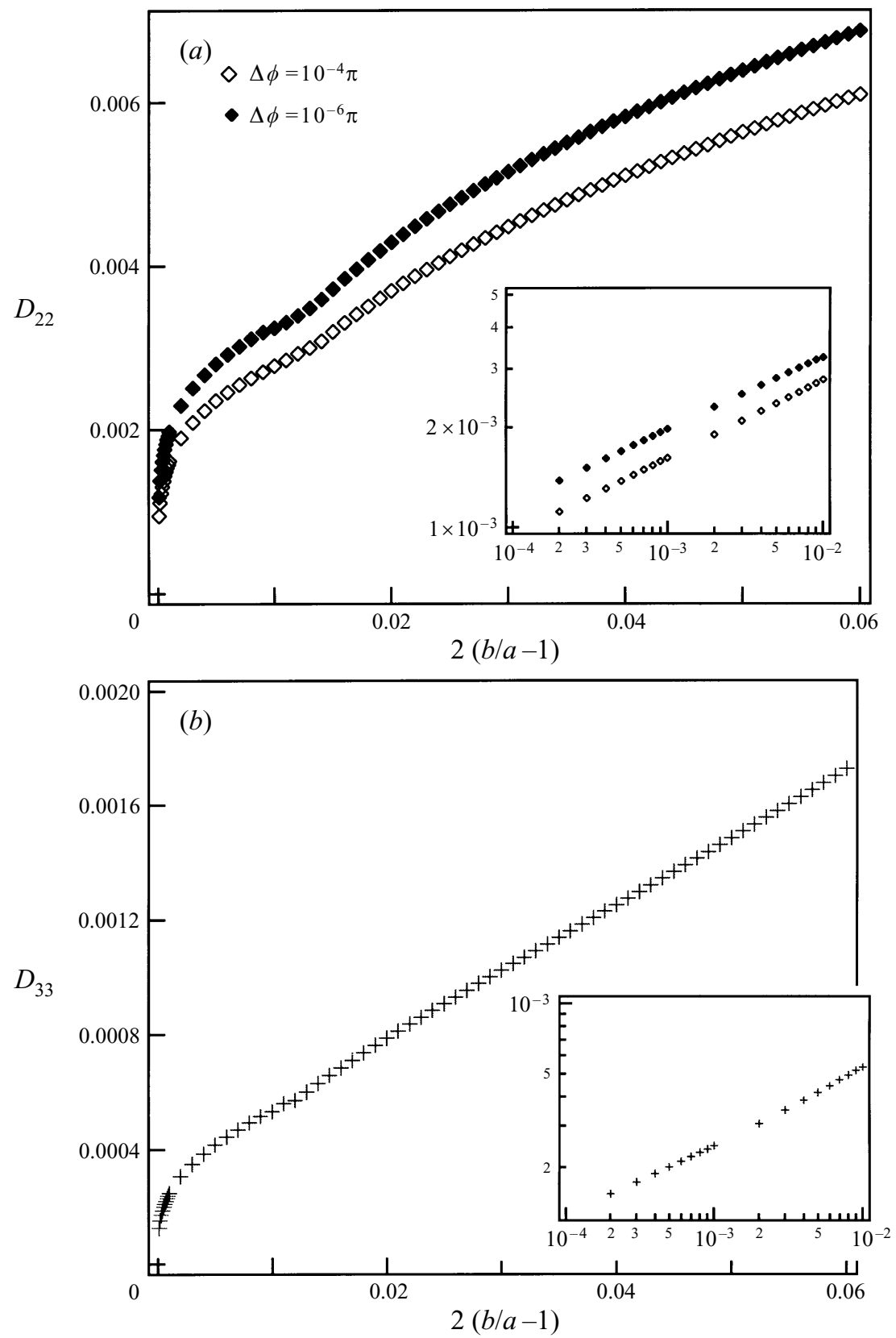

FIGURE $5(a, b)$. For caption see facing page.

for both $\boldsymbol{d}$ and $g$ is $O\left(a \overline{P e^{-1}}\right)$. Third-particle effects will have a quantitative, but not qualitative, influence on the structure of the boundary layer at large $\phi$. Thus, the boundary-layer problem is essentially the same as in the dilute case, with $\overline{P e}$ replacing $P e$, and $g$ asymptotically approaching $g^{\infty}(2 \hat{b} ; \phi)$. Hence, $\boldsymbol{d}$ will be $O(1)$ with respect to both $(\hat{b}-1)$ and $\overline{P e}$ at large $\phi$. From the expression (42) for $\boldsymbol{D}_{\infty}^{s}$, and keeping only 


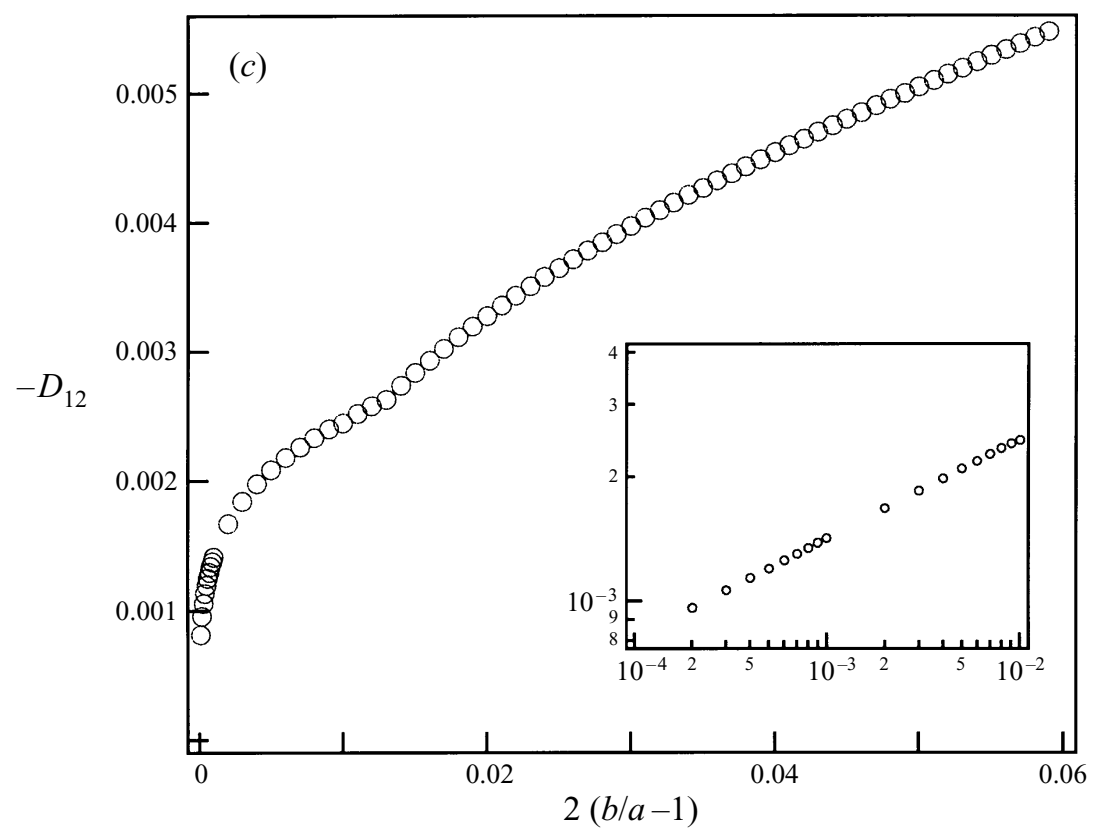

FIGURE 5 . The long-time self-diffusivity components scaled by $\dot{\gamma} a^{2} \phi:(a)$ the diffusivity component in the velocity-gradient direction, $D_{22}$, for $10^{-4} \pi$ (open symbols) and $10^{-6} \pi$ (filled symbols) removed from the polar integration at the divergent endpoint; $(b)$ the diffusivity component in the vorticity direction, $D_{33}$; and $(c)$ the off-diagonal diffusivity component $D_{12}$. Note that $D_{11}=D_{22}$, and that $D_{12}$ is negative and is plotted as $-D_{12}$. The components $D_{i 3}$ and $D_{3 i}, i \neq 3$, are zero.

the dominant contribution in $(\hat{b}-1)$ from the dilute analysis, we have

$$
\begin{aligned}
\boldsymbol{D}_{\infty}^{s} & \sim-D_{0}^{s}(\phi) \phi \hat{b}^{2} \frac{3}{2 \pi} \oint_{r=2 \hat{b}}\left(\int_{0}^{\infty} \boldsymbol{U}^{\prime} \boldsymbol{d} g \mathrm{~d} y\right) \mathrm{d} \Omega \\
& \sim \dot{\gamma} a^{2}(b / a-1)^{0.22} \phi g^{\infty}(2 \hat{b} ; \phi) .
\end{aligned}
$$

Provided there is microstructural asymmetry, we see that even with hydrodynamic interactions the displacement in an encounter is still $O(a)$, and the velocity fluctuations remain $O(\dot{\gamma} a)$. The number of asymmetric encounters giving rise to a net random displacement is now $(\hat{b}-1)^{0.22} g^{\infty}(2 \hat{b} ; \phi)$, showing that in the pure hydrodynamic limit the long-time self-diffusivity is, in the absence of any other symmetry-breaking effect, $O(k T / \eta a)$. The similarity of the expressions for the long-time self-diffusivity with and without hydrodynamic interactions rests in the geometric similarity of the particle encounters in the two cases. The fact that the displacement in an encounter remains $O(a)$ independent of $\phi$ is clearly seen in figures 1 and 2 . There are no particle pairs at contact in the extensional quadrants of the flow: a particle colliding with a reference particle always departs with an $O(a)$ displacement even in a concentrated suspension.

Owing to boundary-layer separation, asymmetry in the microstructure will be present throughout the bulk, and there will be $O(1)$ contributions to the random displacement of a particle from encounters that do not lie in the boundary layer. Unless these displacements are negatively correlated with those in the boundary layer, so as to decrease the boundary-layer contribution, they will only add to the diffusion. It is unlikely that these 'bulk' contributions diverge faster than $g^{\infty}(2 \hat{b} ; \phi)$ at high 
concentrations, so that the boundary-layer estimate should give the correct scaling for the self-diffusivity for all concentrations.

This scaling result predicts that the long-time self-diffusivity diverges as $g^{\infty}(\hat{b} ; \phi)$ as the maximum volume fraction associated with $g^{\infty}$ is approached. A $\boldsymbol{D}_{\infty}^{s}$ that grows with concentration is unusual, but results here from the fact that regardless of the concentration, relative shearing motion takes place at a constant shear rate (although requiring an increasing shear stress to sustain the motion) driving particle encounters with an $O(\dot{\gamma} a)$ velocity fluctuation and an $O(a)$ displacement; the frequency of such encounters increases with increasing concentration and gives rise to the increasing long-time self-diffusivity. The available experimental data on the shear-induced selfdiffusivity of non-Brownian particles (Phan \& Leighton 1997; Phung 1993) show a self-diffusivity that is $O(\phi)$ for small concentrations and increases with increasing $\phi$. However, the simulation and experimental data show a self-diffusivity that is approximately independent of $\phi$ for $0.3<\phi<0.5$, with a value between 0.05 and 0.1 $\dot{\gamma} a^{2}$, in disagreement with our prediction. For the numerical results this difference may be due to the fact that the simulations are of Brownian hard spheres for increasing $P e$, and the infinite-Pe limit may not have been reached. According to our analysis of the pure-hydrodynamic limit the diffusive motion should scale as $k T / \eta a$ rather than as $\dot{\gamma} a^{2}$, and the 'constant' values reported by Phung (1993) for $P e \approx 10^{4}$ may represent a turnover from a growing $\dot{\gamma} a^{2}$ diffusivity to a shear-enhanced Brownian diffusivity. Recent simulations of non-Brownian particles with repulsive interparticle forces (Yurkovetsky 1996) do show a shear-induced self-diffusivity that continues to grow up to $\phi=0.5$, the largest volume fraction simulated.

From (50) and (33), we see that the predicted divergence of $\boldsymbol{D}_{0}^{s}$ as $\phi \rightarrow \phi_{m}$ is smaller than that of the hydrodynamic stress by a factor of $D_{0}^{s}(\phi) \approx\left(1-\phi / \phi_{m}\right)$. This is in keeping with the idea of diffusion due to stress gradients. As we remarked at the end of $\S 6.1$, the diffusivity can also be viewed as the product of the particle mobility times the osmotic pressure gradient force. The pressure gradient scales as $g^{\infty} / \hat{D}_{0}^{s}$, while the relevant mobility is proportional to the short-time self-diffusivity $\hat{D}_{0}^{s}$. It is the short-time self-diffusivity because the test particle is subjected to an infinitesimal force (compared to the shearing force) and does not disturb the microstructure as it moves down its concentration gradient.

\section{Summary and concluding remarks}

We have analysed the influence of weak Brownian motion and repulsive interparticle forces of hard-sphere type on the pair-distribution function in a suspension. In strong flow, i.e. at $P e \gg 1, g$ varies rapidly in a narrow $O\left(a P e^{-1}\right)$ boundary layer near particle contact. In the case of hard spheres subject only to hydrodynamic interactions, Brownian motion renders the contact value of $g$ finite, with $g(2)=O\left(P e^{0.78}\right)$. Despite the fact that this large $g$ lacks fore-aft symmetry, its magnitude is not quite large enough to generate normal stress differences in the pure-hydrodynamic limit because the product of $g(2)$ and the $O\left(a^{3} P e^{-1}\right)$ boundary-layer volume is $O\left(P e^{-0.22}\right)$ and thus vanishes as $P e \rightarrow \infty$.

When the effective radius of a particle is $b>a$, as when particles interact by a strong repulsive force, we have shown through a boundary-layer analysis that $g(\boldsymbol{r})$ is $O(P e)$ in the compressional quadrants and $O(1)$ in the extensional quadrants. Thus, the product of the asymmetry and the boundary-layer volume is finite as $P e \rightarrow \infty$, and normal stress differences scaling as $\eta \dot{\gamma} \phi^{2}$ and a shear-induced self-diffusivity scaling 
as $\dot{\gamma} a^{2} \phi$ are predicted in this limit. We find that as $b / a-1 \rightarrow 0$, the asymmetric portion of $g$ depends on the separation as $(b / a-1)^{0.22}$, and hence the normal stress differences vanish as expected when $b \equiv a$. The exponents of $(b / a-1)^{0.22}$ and $P e^{-0.22}$ are given by a combination of the contact values of hydrodynamic functions,

$$
1+\frac{W(2 a)}{2 A^{\prime}(2 a)}=1+\delta=0.22
$$

where $\delta$ arose in the solution of the similarity problem for $g$ in the case $b \equiv a$.

The results of our analysis help to explain the observation of non-Newtonian behaviour in non-colloidal suspensions. Abundant evidence, some of which is detailed in the Introduction, that the microstructure at large $P e$ is markedly asymmetric in sheared suspensions may now be understood in qualitative and quantitative terms. Both of the cases studied show a large asymmetry, and the slow decay of the influence of this asymmetry upon the rheology, either as $P e \rightarrow \infty$ for $b \equiv a$ or as $b / a-1 \rightarrow 0$ for $P e^{-1}=0$, indicates that, due to the always-present weak Brownian motion and irreversible surface interactions caused by roughness or a finite-ranged force, we may expect to find measurable non-Newtonian effects.

Colloidal dispersions of particles interacting as hard spheres or through repulsive interparticle forces have a low-shear-rate Newtonian viscosity followed by a region of shear thinning before attaining a second, high-shear-rate, viscosity plateau at Péclet numbers of $O(100)$. For hard spheres, the low-shear-rate viscosity is observed to diverge with increasing volume fraction as $\left(1-\phi / \phi_{m}\right)^{-2}$, with the maximum packing corresponding to random close packing $\phi_{m} \approx 0.64$ (Russel et al. 1989). As shown by Brady (1993b), this divergence is due to the Brownian contribution to the stress, which scales as $\eta \dot{\gamma} \phi^{2} g^{e q}(2 ; \phi) / \hat{D}_{0}^{s}(\phi) ; g^{e q}(2, \phi)$ arises because of the contact integral of the Brownian stress (equation (11)), and $\hat{D}_{0}^{s}(\phi)$ appears because the appropriate Péclet number is $\overline{P e}$. At the high-shear-rate viscosity plateau the viscosity is also observed to diverge as $\left(1-\phi / \phi_{m}\right)^{-2}$, but now at a larger maximum packing fraction of $\phi_{m} \approx 0.71$ (Russel et al. 1989). The form of this divergence is suggested by our boundary-layer analysis, (33), with both $g^{\infty}(2 \hat{b} ; \phi)$ and $1 / \hat{D}_{0}^{s}(\phi)$ diverging at the higher volume fraction. Note however, that in order for this divergence to occur there must be interparticle forces. In the pure-hydrodynamic limit the Brownian stress remains $O\left(k T / a^{3}\right)$ and the hydrodynamic stress in this limit does not diverge with an exponent of -2 . The Stokesian Dynamics simulations of Phung et al. (1996) for hard spheres show this second viscosity plateau with the viscosity arising from the hydrodynamic stress; this hydrodynamic viscosity is equal to the high-frequency dynamic viscosity, which diverges with an exponent of -1 .

At Péclet numbers greater than $O(100)$ colloidal dispersions are observed to shear thicken. This thickening may also be understood from our boundary-layer analysis. We saw in $\S 4.2$ that the hydrodynamic contribution to the stress from the boundary layer shear thickens as the boundary layer thins and the $O(P e)$ number of particles are pushed closer to contact. Indeed, even in the complete absence of interparticle forces at $O\left(\phi^{2}\right)$ the shear thickening is evident: at low shear rates the hydrodynamic stress is determined with $g(\boldsymbol{r})=1$ and gives a viscosity contribution of $5 \phi^{2}$. The Brownian viscosity contribution is approximately $0.91 \phi^{2}$. As the Péclet number increases the suspension first shear thins as the Brownian viscosity decreases, and then the pairdistribution function evolves to that given by $p(r)$, with its singular value at contact 
(ignoring the difficulty of regions of closed streamlines), which gives a hydrodynamic viscosity of $6.95 \phi^{2}$.

Although this shear thickening is definitely present at the level of two particles, it may not be sufficient to explain the shear thickening seen experimentally. It has been proposed (Bossis \& Brady 1989) and observed experimentally (D'Haene, Mewis \& Fuller 1993; Bender \& Wagner 1995) that shear thickening is associated with the formation of large aggregates or clusters of particles. The hydrodynamic stress of an aggregate grows as its size cubed, and as the Péclet number increases the aggregate size increases leading to shear thickening. The buildup of particles in the boundary layer near contact is a form of hydrodynamic clustering, but precisely how this twoparticle phenomenon is related to the formation of large clusters and their resultant contribution to the stress is not immediately clear. The common link may be through the increased relative radial velocity at contact and the unknown contact value of $g^{\infty}(2 \hat{b} ; \phi)$, problems that remain to be solved.

This contact value of the pair-distribution function was also seen to play an important role in determining the shear-induced long-time self-diffusivity, as it gives the number of particle encounters with velocity fluctuation $\dot{\gamma} a$ and displacement $a$; i.e. $D_{\infty}^{s} \sim \dot{\gamma} a^{2}(\hat{b}-a)^{0.22} \phi g^{\infty}(2 \hat{b} ; \phi)$. Furthermore, the hard-sphere repulsive force and singular influence of Brownian motion are critical for obtaining a diffusive behaviour - they are the underlying origin of irreversibility.

It should also be evident from this boundary-layer phenomenon at high Péclet number that the numerical simulation of suspension behaviour for Péclet numbers in excess of several thousand is a very difficult problem. In order to properly resolve the boundary layer, exceedingly small time steps will be necessary to faithfully follow particle trajectories near contact and resolve the dominant physics occurring there.

Although we have only considered a repulsive force of hard-sphere type, this was done for convenience in formulating the boundary-value problems to be solved. The same behaviour will be obtained with a repulsive force of extended (but short) range, even in the complete absence of Brownian motion as shown in \$3.2. A boundary layer at 'contact' whose thickness will depend on the ratio of the amplitude of the shear to repulsive forces will be set up at high shear rates with the same structure and consequences as that studied here.

The results presented here for the presence of normal stresses as $P e \rightarrow \infty$ may be important in understanding the phenomena of shear-induced particle migration in inhomogeneous shear flows. Nott \& Brady (1994) have advanced the theory that normal stresses, rather than variations in shear rate, cause migration. They showed that no migration would occur in torsional flow, as is found experimentally (Chow et al. 1994), if $2 \Sigma_{r r}=\Sigma_{\theta \theta}$. Interestingly, the normal stresses predicted in $\$ 3.2$ show precisely this relationship. Further, we have shown here that the shearinduced diffusive motion can be directly related to stress driving forces, a result postulated by Nott \& Brady (1994). Finally, the singular nature of the boundarylayer problem is responsible for the microstructural asymmetry that underlies the form of the constitutive equations postulated by Nott \& Brady (1994), and the results of this analysis can be used to determine the volume-fraction dependence of these constitutive equations.

Although the present study is devoted to microstructure in linear flows, it is worth noting that the high-Péclet-number boundary-layer problem was suggested in a study by Batchelor (1982) of the microstructure of sedimenting particles. Batchelor \& Wen (1982) applied the theory of Batchelor (1982) to numerically evaluate $g(\boldsymbol{r})$ in a dilute large- $P e$ sedimentation problem. These authors did not resolve the solution for $g$ 
up to contact and thus were unable to determine the manner in which the singular contact value of $g$ is made finite by Brownian motion. It would be of interest to apply the methods described here to the sedimentation problem.

This work was supported in part by grant No. CTS-9420415 from the National Science Foundation and by grant No. N00014-95-1-0423 from the Office of Naval Research. The authors wish to thank Francis Gadala-Maria for providing figure 2. J.F.B. wishes to thank the Isaac Newton Institute for Mathematical Sciences, Cambridge University, for their hospitality during the writing of this paper.

\section{Appendix. Similarity solution: no interparticle forces}

A change of variables in $(36 a)$ to $\zeta=-\xi$ yields the governing equation for $u(\zeta)$,

$$
\zeta u^{\prime \prime}+(1-\zeta) u^{\prime}+\delta u=0,
$$

the standard form which has the general solution (Abramowitz \& Stegun 1972, chap. 13)

$$
u(\zeta)=\lambda_{1} M(-\delta, 1, \zeta)+\lambda_{2} U(-\delta, 1, \zeta)
$$

where $M$ and $U$ are confluent hypergeometric functions, and $\lambda_{1}$ and $\lambda_{2}$ are arbitrary constants. For second argument of unity, $U$ is logarithmically singular at $\zeta=0$ and is therefore discarded. The argument $\zeta$ is negative, and

$$
M(-\delta, 1, \zeta) \sim K(-\zeta)^{\delta}=K \eta^{\delta} \quad \text { as } \quad \eta \rightarrow \infty,
$$

with $K$ a constant. This far-field form of $M$ agrees with the asymptotic form of $u$ given by (39) that was shown to satisfy the boundary condition (36c) of matching the radial dependence of $p$. The condition $(36 b)$ at contact is satisfied because $M^{\prime}(-\delta, 1,0)=-\delta$ and thus the product $\zeta M^{\prime}(-\delta, 1, \zeta)$ vanishes at $\zeta=0$.

To complete the solution for $g$ within the boundary layer, we determine the scaling function $Y(\theta, \varphi)$, which has the physical meaning of a variable boundarylayer thickness. It is sufficient, due to symmetry of the pair configuration and bulk flow, to determine $Y$ in the restricted domain $0<\theta<\pi / 2$ and $3 \pi / 4<\varphi<5 \pi / 4$. The solution elsewhere may be obtained from the symmetry relations

$$
Y(\pi-\theta, \varphi)=Y(\theta, \varphi), \quad Y(\theta, \varphi)=Y(\theta, \varphi+\pi), \quad \text { and } \quad Y(\theta, \varphi)=Y(\theta, 3 \pi / 2-\varphi) .
$$

Two conditions on $Y$ are required, which we choose to be finiteness of $Y$ at $\varphi=3 \pi / 4$ and $\partial Y / \partial \theta=0$ at $\theta=\pi / 2$.

The solution to (37) is found by the method of characteristics (Carrier \& Pearson 1988). Rewriting (37) as

$$
\frac{\partial Y}{\partial \varphi}+v(\theta, \varphi) \frac{\partial Y}{\partial \theta}=w(Y, \theta, \varphi)
$$

where

$$
v(\theta, \varphi)=\frac{\gamma_{\theta}}{\gamma_{\varphi}} \sin \theta, \quad \text { and } \quad w(Y, \theta, \varphi)=4 \frac{\sin \theta}{\gamma_{\varphi}} \frac{1-\gamma_{r} Y}{1-B(2)},
$$

we may interpret $w$ as the complete derivative $w=\mathrm{d} Y / \mathrm{d} s$ with $\mathrm{d} \varphi / \mathrm{d} s=1$ and $\mathrm{d} \theta / \mathrm{d} s=v$. For $\varphi=3 \pi / 4,(37)$ reduces to

$$
\frac{\mathrm{d} Y(\theta, 3 \pi / 4)}{\mathrm{d} \theta}+\left(k_{1} \tan \theta\right) Y=-k_{2} \sec \theta \operatorname{cosec} \theta,
$$


with solution

$$
Y(\theta, 3 \pi / 4)=k_{2}(\cos \theta)^{k_{1}} \int_{\theta}^{\pi / 2}(\cos x)^{-\left(1+k_{1}\right)} \operatorname{cosec} x \mathrm{~d} x
$$

where

$$
k_{1}=\frac{2 A^{\prime}(2)}{1-B(2)} \approx-13.7, \quad \text { and } \quad k_{2}=\frac{4}{1-B(2)} \approx 6.7 .
$$

Similarly, for $\theta=\pi / 2$, (37) reduces to

$$
\frac{\mathrm{d} Y(\pi / 2, \varphi)}{\mathrm{d} \varphi}+\left(k_{1} \tan 2 \varphi\right) Y=k_{2} \operatorname{cosec} 2 \varphi,
$$

with solution

$$
Y(\pi / 2, \varphi)=k_{2}(\cos 2 \varphi)^{k_{1} / 2} \int_{3 \pi / 4}^{\varphi}(\cos 2 x)^{-\left(1+k_{1} / 2\right)} \mathrm{d} x
$$

\section{REFERENCES}

Abramowitz, M. \& Stegun, I. A. 1972 Handbook of Mathematical Functions. Dover.

BATCHELOR, G. K. 1977 The effect of Brownian motion on the bulk stress in a suspension of spherical particles. J. Fluid Mech. 83, 97.

BatcheloR, G. K. 1982 Sedimentation in a dilute polydisperse system of interacting spheres. Part 1. General theory. J. Fluid Mech. 119, 379.

Batchelor, G. K. \& Green, J. T. $1972 a$ The hydrodynamic interactions of two small freely-moving spheres in a linear flow field. J. Fluid Mech. 56, 375.

Batchelor, G. K. \& Green, J. T. 1972b The determination of the bulk stress in a suspension of spherical particles to order $c^{2}$. J. Fluid Mech. 56, 401.

BAtCheloR, G. K. \& WeN, C. S. 1982 Sedimentation in a dilute polydisperse system of interacting spheres. Part 2. Numerical results. J. Fluid Mech. 124, 495 (and Corrigendum J. Fluid Mech. 137 (1983), 467).

Bender, J. W. \& Wagner, N. J. 1995 Optical measurement of the contribution of colloidal forces to the rheology of concentrated suspensions J. Colloid Interface Sci. 172, 171.

Berne, B. J. \& PecorA, R. 1976 Dynamic Light Scattering. Wiley.

Bossis, G. \& Brady, J. F. 1989 The rheology of Brownian suspensions. J. Chem. Phys. 91, 1866.

Bossis, G., Brady, J. F. \& Mathis, C. 1988 Shear-induced structure in colloidal suspensions. I. Numerical simulations. J. Colloid Interface Sci. 126, 1.

Brady, J. F. 1993a Brownian motion, hydrodynamics and the osmotic pressure. J. Chem. Phys. 98, 3335.

Brady, J. F. $1993 b$ The rheological behaviour of concentrated colloidal dispersions. J. Chem. Phys. 99, 567.

Brady, J. F. 1994 The long-time self-diffusivity in concentrated colloidal dispersions. J. Fluid Mech. 272, 109

Brady, J. F. \& Bossis, G. 1985 The rheology of concentrated suspensions in simple shear by numerical simulation. J. Fluid Mech. 155, 105.

Brady, J. F. \& Bossis, G. 1988 Stokesian Dynamics. Ann. Rev. Fluid Mech. 20, 111.

Brady, J. F. \& Vicic, M. A. 1995 Normal stresses in colloidal dispersions. J. Rheol. 39, 545.

Carrier, G. F. \& Pearson, C. E. 1988 Partial Differential Equations. Academic.

Chow, A. W., Sinton, S. W., Iwamiya, J. H. \& Stephens, T. S. 1994 Shear-induced migration in Couette and parallel-plate viscometers: NMR imaging and stress measurements. Phys. Fluids A 6, 2561.

Cunha, F. R. DA \& Hinch, E. J. 1996 Shear-induced dispersion in a dilute suspension of rough spheres. J. Fluid Mech. 309, 211.

D’Haene, P., Mewis, J. \& Fuller, G. G. 1993 Scattering dichroism measurements of flow-induced structure of a shear thickening suspension. J. Colloid Interface Sci. 156, 350. 
Eckstein, E. C., Bailey, D. G. \& Shapiro, A. H. 1977 Self-diffusion of particles in shear flow of a suspension. J. Fluid Mech. 79, 191.

Gadala-Maria, F. 1979 The rheology of concentrated suspensions. PhD thesis, Stanford University.

Gadala-Maria, F. \& Acrivos, A. 1980 Shear-induced structure in a concentrated suspension of solid spheres. J. Rheol. 24 (6), 799.

Hansen, J. P. \& McDonald, I. R. 1986 Theory of Simple Fluids. Academic.

Jeffrey, D. J., Morris, J. F. \& Brady, J. F. 1993 The pressure moments for two spheres in a low-Reynolds-number flow. Phys. Fluids A 5, 2317.

Jones, R. B. \& Burfield, G. S. 1982 Memory effects in the diffusion of an interacting polydisperse suspension. Part 1. Physica A 111, 562.

Kim, S. \& Karrila, S. J. 1991 Microhydrodynamics: Principles and Selected Applications. Butterworth-Heinemann.

Morris, J. F. \& Brady, J. F. 1996 Self-diffusion in sheared suspensions. J. Fluid Mech. 312, 223.

Nott, P. \& Brady, J. F. 1994 Pressure-driven flow of suspensions: simulation and theory. J. Fluid Mech. 275, 157.

PARsi, F. \& GAdAla-Maria, F. 1987 Fore-and-aft asymmetry in a concentrated suspension of solid spheres J. Rheol. 31, 725.

Phan, S. \& Leighton, D. T. 1997 Measurement of the shear-induced tracer diffusivity in concentrated suspensions. J. Fluid Mech. (to appear)

Phung, T. N. 1993 Behaviour of concentrated colloidal dispersions by Stokesian dynamics. PhD thesis, California Institute of Technology.

Phung, T. N., Brady, J. F. \& Bossis, G. 1996 Stokesian Dynamics simulation of Brownian suspensions. J. Fluid Mech. 313, 181.

Pusey, P. N. 1991 Colloidal suspensions. In Liquids, Freezing, and Glass Transition (ed. J. P. Hansen, D. Levesque \& J. Zinn-Justin). Elsevier.

Rallison, J. M. \& Hinch, E. J. 1986 The effect of particle interactions on dynamic light scattering from a dilute suspension. J. Fluid Mech. 167, 131.

Russel, W. B., Saville, D. A. \& Schowalter, W. R. 1989 Colloidal Dispersions, Ch. 14. Cambridge University Press.

Smart, J. R. \& Leighton, D. T. 1989 Measurement of the hydrodynamic surface roughness of noncolloidal spheres. Phys. Fluids A 1, 52.

Thomas, D. G. 1965 Transport characteristics of suspensions. VIII A note on the viscosity of Newtonian suspensions of uniform spherical particles. J. Colloid Interface Sci. 20, 267.

YURKOVETSKY, Y. 1996 I. Statistical mechanics of bubbly liquids. II. Behaviour of sheared suspensions of non-Brownian particles. $\mathrm{PhD}$ thesis, California Institute of Technology. 